Aus der Abteilung Neurochirurgie

(Prof. Dr. med. V. Rohde)

im Zentrum Neurologische Medizin

der Medizinischen Fakultät der Universität Göttingen

\title{
Nichtinvasiv neuronavigierte transkranielle Dopplersonographie
}

\author{
INAUGURAL-DISSERTATION \\ zur Erlangung des Doktorgrades \\ der Medizinischen Fakultät \\ der Georg-August-Universität zu Göttingen
}

vorgelegt von

CHRISTIAN GREKE

aus Vechta

Göttingen 2011 
Dekan:

1. Berichterstatter:

2. Berichterstatter:

Tag der mündlichen Prüfung:
Prof. Dr. med. C. Frömmel

Prof. Dr. med. A. Giese

PD Dr. med. F. Mielck 


\section{Inhalt}

1 Einleitung ........................................................................................................................................................ 1

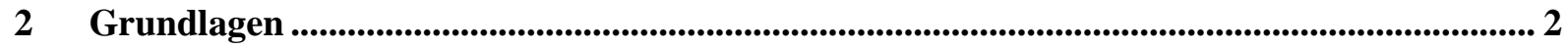

2.1 Grundlagen der Dopplersonographie - Physik und Historie ................................................. 2

2.2 Grundlagen der transkraniellen Dopplersonographie........................................................... 4

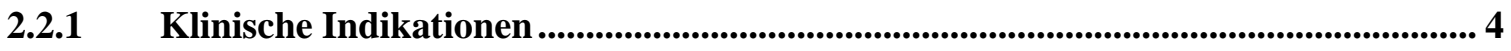

2.2.1.1 Vasospasmusmonitoring nach subarachnoidalen Hirnblutungen ................................ 4

2.2.1.2 Weitere Anwendungen ................................................................................................................. 5

2.2.2 Transkranielle Dopplersonographie in der klinischen Anwendung ............................ 5

2.2.2.1 Problematik der Schallabsorption ..................................................................................... 6

2.2.2.2 Konventionelle TCD-Untersuchung .......................................................................................... 8

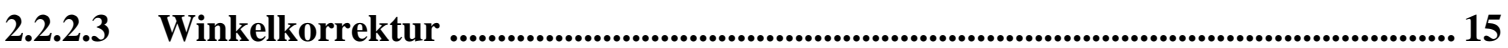

2.2.3 Transkranielle Duplexsonographie........................................................................... 16

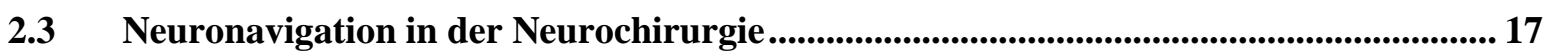

2.3.1 Technische Grundlagen und präoperatives Vorgehen............................................ 17

2.3.2 Genauigkeit der Neuronavigation.......................................................................................... 19

2.3.3 Klinische Anwendung in der Neurochirurgie ............................................................... 19

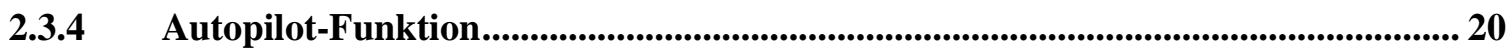

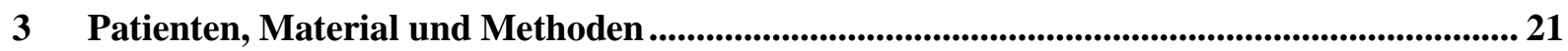

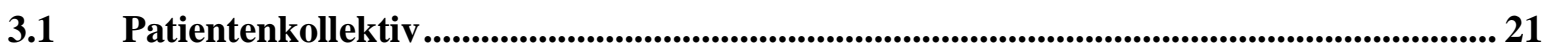

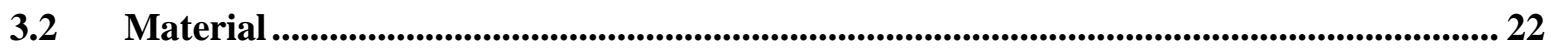

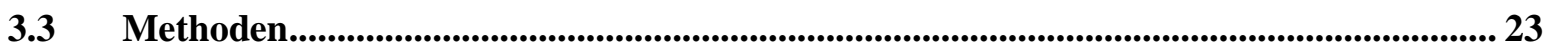

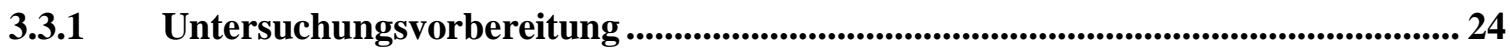

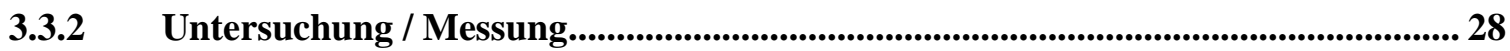

3.3.3 Datenauswertung / Statistik......................................................................................... 30

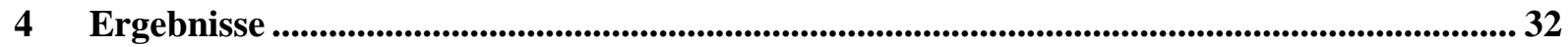

4.1 Identifikation von spezifischen Gefäßsegmenten ................................................................... 32

4.2 Räumliche Genauigkeit..................................................................................................................... 35

4.3 Genauigkeit im Verlauf der Untersuchung.................................................................................. 39

4.4 Abhängigkeit der Ergebnisse vom Alter des CT-A-Datensatzes......................................... 41

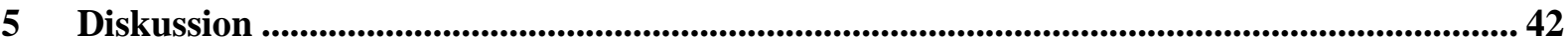

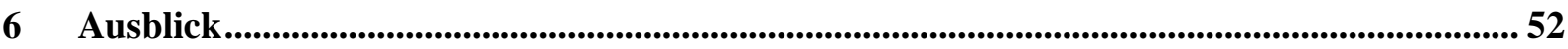

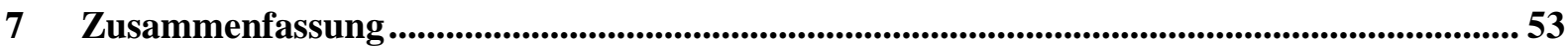




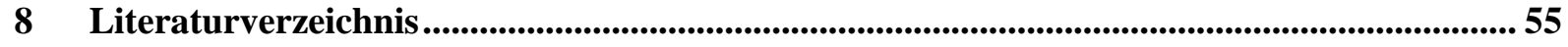

9 Anhang / Publikationen ..................................................................................................................60

9.1 Image Guidance for Transcranial Doppler Ultrasonography................................................ 60

9.2 Image-guided transcranial Doppler sonography for monitoring of defined segments of

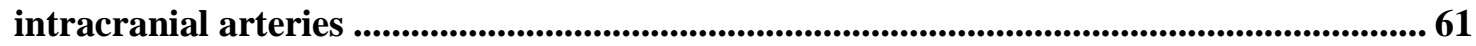




\section{Abkürzungsverzeichnis}

A.

Aa.

ACA

ACC

ACOM

BA

BA-Kopf

c

CT

CT-A

DID

f

$\mathrm{Hz}$

ICA

Kap.

Karotis-T

MCA

mean

MR-A

MRT

MultiDop

NaviTCD

PCA

PCOM

SA

$\mathrm{SAB}$

Target

TCCD

TCD

UMG
Arteria

Arteriae

Arteria cerebri anterior

Arteria carotis communis

Arteria communicans anterior

Arteria basilaris

Bifurkation der Arteria basilaris

Ausbreitungsgeschwindigkeit

Computertomographie

Computertomographische Angiographie

verzögertes neurologisches Defizit

Frequenz

Hertz

Arteria carotis interna

Kapitel

Bifurkation der A. carotis interna

Arteria cerebri media

Mittelwert

Magnetresonanztomographische Angiographie

Magnetresonanztomographie

DWL MultiDop digitalTM

neuronavigierte transkranielle Dopplersonographie

Arteria cerebri posterior

Arteria communicans posterior

Standardabweichung

subarachnoidale Hirnblutung

Navigationszielpunkt

transkranielle Duplexsonographie

transkranielle Dopplersonographie

Universitätsmedizin Göttingen 
V

VA
Geschwindigkeit

Arteria vertebralis 


\section{Einleitung}

Schnell nach Aaslids Erstbeschreibung der transkraniellen Dopplersonographie (TCD) im Jahr 1982, mit der nicht-invasiv mittels Ultraschall die Blutflussgeschwindigkeit in den basalen Hirnarterien bestimmt werden kann (Aaslid et al. 1982), hat sich diese Methode für verschiedene Fragestellungen im klinischen Alltag etabliert (Singh et al. 2001).

Da einige Faktoren, wie beispielsweise die Routine und der Ausbildungsstand des Untersuchers die Anwendung hinsichtlich der Ergebnisverwertbarkeit limitieren (von Reutern 1982, Kremser et al. 1999), stellte sich die Frage, ob der Schwierigkeitsgrad der Methode durch die Erweiterung der TCD um eine Bildgebung verringert werden könnte. Im Gegensatz zur Duplexsonographie handelt es sich bei der Dopplersonographie nicht um eine Schnittbildtechnik, sodass die Identifikation des insonierten Gefäßes nicht bildgeführt geschieht, sondern mithilfe von Flusscharakteristik des Blutes, Einschalltiefe und -richtung sowie extrakranieller Gefäßkompressionstests erfolgen muss. Letzteres birgt zusätzliche Fehlerquellen (von Reutern und Büdingen 1993) und ist im Falle des KarotisKompressionstests auch mit Risiken behaftet (Khaffaf et al. 1994). Auf Einzelheiten und Zusammenhänge der oben genannten Probleme soll im Kapitel 2.2.2 genauer eingegangen werden.

Die dieser Arbeit zugrunde liegende Idee war es, die konventionelle transkranielle Dopplersonographie durch Kombination mit einem Neuronavigationsverfahren zu erweitern, die Genauigkeit dieser Kombination zu überprüfen (vgl. S. 35, Abb. 14) und zu evaluieren, ob die anatomische Orientierung an Navigationsschnittbildern eine Hilfestellung für die klinische Anwendung sein kann. 


\section{Grundlagen}

\subsection{Grundlagen der Dopplersonographie - Physik und Historie}

Physikalische Grundlage für die Messung von Blutflussgeschwindigkeiten mittels Ultraschall ist der sogenannte Dopplereffekt, der 1842 von Christian Johann Doppler (1803-1853), einem österreichischen Mathematiker und Physiker, in seinem Buch „Ueber das farbige Licht der Doppelsterne und einiger anderer Gestirne des Himmels“" beschrieben wurde (Doppler 1842). Doppler stellte fest, dass es durch die Relativbewegung zwischen einer Lichtquelle (in diesem Fall das Licht der Sterne) und dem Beobachter zu einer Frequenzänderung des ausgesendeten Lichts kommt. So werde die Frequenz bei Bewegung eines Objektes auf den Beobachter/Empfänger zu kleiner, und werde größer, wenn sich das Objekt von diesem entferne (Doppler 1842).

Aus der Differenz der ausgesendeten und der empfangenen Frequenz des Schalls ergibt sich der sogenannte Dopplershift. Aus der Kenntnis des Dopplershifts, der ausgesendeten Schallfrequenz, der Schallausbreitungsgeschwindigkeit und der Voraussetzung, dass sich das Objekt in einem Winkel von $0^{\circ}$ zum Empfänger befindet, lässt sich die Geschwindigkeit des Objektes bestimmen. Da im Falle der Dopplersonographie die Sonde gleichzeitig als Sender und Empfänger dient, hat der Schall eine doppelte Laufzeit. Die Erythrozyten des Blutes dienen hierbei als Reflektor des von einer Sonde ausgestrahlten Ultraschallstrahls, was den Faktor 2 im Nenner der folgenden Formel erklärt:

$\mathrm{v}=\Delta \mathrm{f} * \mathrm{c} / 2 * \mathrm{f} \quad(\mathrm{v}=$ Geschwindigkeit; $\mathrm{f}=$ Frequenz; $\mathrm{c}=$ Ausbreitungsgeschwindigkeit $)$.

Nutzt man diese Kenntnis für die Messung der Blutflussgeschwindigkeit des Blutes in den basalen Hirnarterien, so ergibt sich die folgende Formel, da für c im menschlichen Hirngewebe $1560 \mathrm{~m} / \mathrm{s}$ angenommen werden kann und für die transkranielle 
Dopplersonographie typischerweise $2 \mathrm{MHz}-$ Sonden verwendet werden (Diehl und Berlit 1996).

$\mathrm{v}=39 * \Delta \mathrm{f} \quad(\mathrm{v}=$ Geschwindigkeit in $\mathrm{cm} / \mathrm{s} ; \Delta \mathrm{f}=$ Frequenzshift in $\mathrm{kHz})$

Ein Frequenzshift von $1 \mathrm{kHz}$ entspricht also einer Geschwindigkeit von ca. $39 \mathrm{~cm} / \mathrm{s}$. Zum Vergleich beträgt die normale Blutflussgeschwindigkeit eines Gesunden in der A. cerebri media $62 \pm 12 \mathrm{~cm} / \mathrm{s}$ (Aaslid et al. 1982). Dies entspricht einem Dopplershift von etwa 1,59 $\mathrm{kHz}$

Zunächst blieb die Anwendung der Dopplersonographie in der Medizin auf extrakranielle Anwendungen beschränkt, so dass eine Beurteilung der intrakraniellen Gefäße lediglich indirekt durch Ableitung der extrakraniellen Arterien möglich war (Keller et al. 1973). 1982 publizierte der Erstbeschreiber der TCD, Rune Aaslid, dass der menschliche Schädelknochen transtemporal an einigen Stellen mit niedrigen Ultraschallsendefrequenzen von 1-2 MHz und höheren Schallenergien $(350 \mathrm{~mW})$ durchdringbar sei. So gelang es ihm, erstmals die Flusscharakteristiken und Flussgeschwindigkeiten der Aa. cerebri media, anterior und posterior direkt darzustellen (Aaslid et al. 1982). In den folgenden Jahren erlangte die Methode breite Akzeptanz für verschiedene Anwendungsgebiete. Dazu zählen das zerebrale Vasospasmusmonitoring, Okklusions- und Stenosierungsdiagnostik, Evaluierung der zerebralen Autoregulation, die nichtinvasive Abschätzung des intrazerebralen Druckes und die Hirntoddiagnostik (Babikian et al. 2000, Tsivgoulis et al. 2009). 


\subsection{Grundlagen der transkraniellen Dopplersonographie}

\subsubsection{Klinische Indikationen}

\subsubsection{Vasospasmusmonitoring nach subarachnoidalen Hirnblutungen}

Eine häufige Komplikation von subarachnoidalen Blutungsereignissen (SAB), die etwa 5\% aller Schlaganfälle ausmachen und in $85 \%$ der Fälle auf rupturierte Aneurysmen zurückzuführen sind (van Gijn et al. 2007), ist die Ausprägung von Gefäßspasmen innerhalb von zwei Wochen nach dem Blutungsereignis. In einem Review von Dorsch und King 1994, in dem mehr als 30.000 Fälle betrachtet wurden, zeigte sich, dass durchschnittlich $67,3 \%$ der Betroffenen im Verlauf nach einer SAB nachweisbare Spasmen entwickelten; 32,5\% bekamen klinisch symptomatische Spasmen oder ein verzögertes neurologisches Defizit (DID), von denen wiederum 30\% verstarben (Dorsch und King 1994). Mit 28,5\% zeigte die Langzeitanalyse dieses Reviews im Zeitraum von 1994 bis 2009 eine etwas geringere Rate von klinisch relevanten Spasmen bzw. DID (Dorsch 2011). Es wird somit ersichtlich, dass post-hämorrhagische intrazerebrale Gefäßspasmen eine der wesentlichsten Komplikationen nach SAB darstellen. Der „Goldstandard“ zur Diagnostik von Vasospasmen ist die arterielle Angiographie, die allerdings bedingt durch ihre Invasivität mit Risiken behaftet ist (Willinsky et al. 2003). Alternativ kann durch nichtinvasives Monitoring von Blutflussgeschwindigkeiten mittels TCD ein Rückschluss auf eine Veränderung des Gefäßkalibers gezogen werden. Dabei steht das Ausmaß der Veränderung der Flussgeschwindigkeit in einer inversen Relation zum Gefäßdurchmesser (Aaslid 2002). Da die TCD nicht-invasiv, beliebig häufig wiederholbar ist, am Patientenbett durchgeführt werden kann und zudem kostengünstig ist, ist sie ein häufig genutztes Monitoringverfahren für regelmäßige, serielle Untersuchungen auf neurochirurgischen Intensivstationen (Rigamonti et al. 2008). 


\subsubsection{Weitere Anwendungen}

Die häufigste Anwendung in der Neurochirurgie findet die transkranielle Dopplersonographie, wie oben beschrieben, bei der Detektion von Vasospasmen nach subarachnoidalen Blutungen (SAB). Weitere klassische Anwendungsbereiche der TCD sind die Evaluierung der cerebralen Autoregulation, Detektion von Gefäßstenosen und Okklusionen, Echt-Zeit-Rekanalisationsmonitoring nach embolischem Gefäßverschluss, intraoperative Embolusdetektion, Rechts-/Linksshunt-Diagnostik und die Hirntoddiagnostik (Tsivgoulis et al. 2009).

Seit einiger Zeit wird ferner die Kombination aus medikamentöser Lysetherapie und Sonothrombolyse bzw. auch die alleinige Sonothrombolyse mittels TCD erforscht. Die Studie von Alexandrov aus dem Jahr 2009 konnte zeigen, dass die Rekanalisationsrate innerhalb der ersten zwei Stunden bei Kombination beider Methoden signifikant höher war. Da bei bisherigen Versuchen aber Komplikationen, wie z.B. Blutungsereignisse, aufgetreten sind, hat diese Methode noch keinen Einzug in die klinische Routine erhalten und wird weiter untersucht (Alexandrov 2009).

\subsubsection{Transkranielle Dopplersonographie in der klinischen Anwendung}

Obwohl sich die TCD im der klinischen Routine schnell etabliert hat, bietet die Methode nicht nur Vorteile sondern auch bedeutende Nachteile, die dem Untersucher bewusst sein müssen, um Probleme, Fehler und Fehlerquellen zu erkennen, auf die in diesem Kapitel näher eingegangen werden soll. 


\subsubsection{Problematik der Schallabsorption}

Erst seit 1982 findet die TCD Anwendung im medizinischen Bereich. Aaslid konnte damals zeigen, dass es möglich ist, den menschlichen Schädelknochen an bestimmten Stellen mittels Ultraschall zu durchdringen und Flussgeschwindigkeiten der basalen Hirnarterien abzuleiten.

Im Folgenden soll auf die gebräuchlichsten Beschallungswege und Probleme des transtemporalen Schallfensters eingegangen werden. Abhängig vom zu untersuchenden Gefäß werden drei grundsätzlich verschiedene Beschallungswege genutzt, um intrakranielle Gefäße abzuleiten (Nicoletto und Burkman 2009).

\begin{tabular}{|c|c|}
\hline Beschallungsweg & $\underline{\text { ableitbare Gefäße }}$ \\
\hline Transtemporales Schallfenster & ACA, MCA, PCA, Endstrecke ICA \\
\hline Transforaminales Schallfenster & VA, BA \\
\hline Transorbitales Schallfenster & ICA im Karotissiphon, A. ophthalmica \\
\hline
\end{tabular}

Tab. 1: TCD Beschallungswege (nach Nicoletto und Burkman 2009)

Dabei unterscheidet sich das transtemporale Schallfenster $($ Schallfenster = Orte am Schädel, die durchlässig für Ultraschall sind) vom transforaminalen und transorbitalen dadurch, dass transtemporal die Temporalschuppe durchdrungen werden muss. Transorbital und transforaminal sind mit der Fissura orbitalis superior bzw. dem Foramen magnum natürliche Knochenöffnungen und somit freier Zugang zu den Gefäßen vorhanden. 


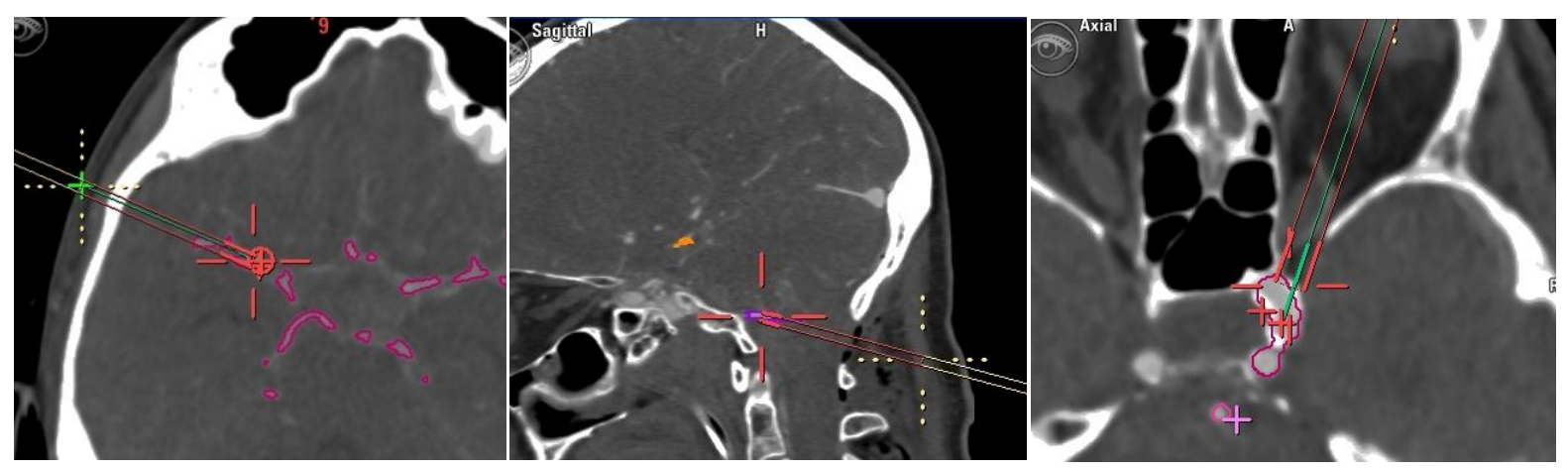

Abb. 1: typische Beschallungswege für TCD-Untersuchungen. Links: transtemporal MCA links. Mitte: transforaminal VA links. Rechts: transorbital ICA

Knochenmatrix stellt allerdings eine physikalische Barriere für Ultraschallwellen dar. Ultraschallwellen werden bei Durchtritt durch den Knochen ebenso gestreut wie auch abgeschwächt (Evans 2006). Bei Verwendung von 2 MHz-Sonden (wie bei TCD heute etabliert) werden $88 \%$ der Sendeenergie von einer 1,4 mm dicken Temporalschuppe absorbiert. Nach Reflektion des Schalls an den Erythrozyten erfolgt erneut eine ähnliche Abschwächung auf dem Rückweg zur Sonde. Somit steht für die Untersuchung intrakranieller Gefäße nur noch ein Bruchteil der ausgesendeten Energie zur Verfügung. Bereits bei einer Knochendicke von 2,5 mm beträgt die einfache Abschwächung 99\% (Grolimund 1986). Hieraus erklärt sich die Problematik, dass für die TCD-Untersuchung nur wenige bzw. in einigen Fällen gar keine transtemporalen Beschallungswege vorhanden sind und dadurch die Erfassung der ACA, MCA und PCA erschwert bzw. unmöglich wird. In der Literatur wurde gezeigt, dass es einen Zusammenhang zwischen dem Fehlen eines Knochenfensters und der Kombination folgender drei Parameter gibt: Knochendicke, Alter und des Geschlecht des Patienten (Wijnhoud et al. 2008). Ferner zeigte sich bei Asiaten und Afroamerikanern eine erhöhte Rate von inadäquaten Schallfenstern (Yagita et al. 1996). Itoh et al. stellten in einer Studie an japanischen Probanden fest, dass lediglich in 77,1\% der Fälle ein Signal der MCA 
ableitbar war. Bei japanischen Frauen (>70 Jahre) lag die Erfolgsquote lediglich noch bei 17,0\% (Itoh et al. 1993). Im Allgemeinen finden sich in der Literatur aber unterschiedliche Angaben. Werte zwischen fünf und zehn Prozent in der europäischen Bevölkerung sind als realistisch einzuschätzen.

Aufgrund dieser Problematik ist nicht selten einige Geduld erforderlich, bis ein Dopplersignal der intrakraniellen Gefäße gefunden werden kann. Von vielen Untersuchern wird zur Schallfenstersuche eine Fokustiefe von 55-60 mm voreingestellt, da in dieser Tiefe am wahrscheinlichsten ein Signal des Circulus Willisi abgeleitet werden kann (Widder und Görtler 2004).

\subsubsection{Konventionelle TCD-Untersuchung}

\section{Normale Anatomie}

Von einer „normalen“ zerebralen Gefäßanatomie kann nur bedingt gesprochen werden, da zahlreiche Varianten existieren. Die häufigste Variante (45,2\% (Kapoor et al. 2008)) soll als Normvariante bezeichnet und im Folgenden kurz beschrieben werden.

Das menschliche Gehirn wird über ein vorderes und ein hinteres Stromgebiet versorgt. Das vordere Stromgebiet wird aus beiden Aa. carotis (ACC) gespeist. Dabei ist die rechte ACC ein Ast des Truncus brachiocephalicus und die linke ACC ein direkter Ast des Aortenbogens. An der sogenannten Karotisbifurkation teilt sich die ACC beidseits in A. carotis externa und A. carotis interna (ICA). Die ICA tritt durch den Canalis caroticus in den Schädel ein und gibt als ersten Ast dort die Arteria ophthalmica ab, die durch das transorbitale Schallfenster beschallt werden kann. Im weiteren Verlauf teilt sich die ICA am Karotis-T in die A. cerebri media (MCA), die von dort nach lateral zieht, und die A. cerebri anterior (ACA), die zur 
Mittellinie verläuft. Nahe der Mittellinie verbindet die A. communicans anterior (ACOM) beide ACA miteinander.

Die beiden Vertebralarterien, die Äste der Aa. subclaviae sind, treten durch das Foramen magnum in den Schädel ein und vereinigen sich im Verlauf zur A. basilaris (BA). Die BA teilt sich am Basilaris-Kopf in die zwei Aa. cerebri posteriores (PCA), die in der Normvariante zuerst nach lateral und im weiteren Verlauf nach okzipital ziehen. Aus dem nach lateral verlaufendem Teil der PCA entspringt die A. communicans posterior (PCOM), die das vordere mit dem hinteren Stromgebiet verbindet und in die ICA unterhalb des KarotisT mündet.
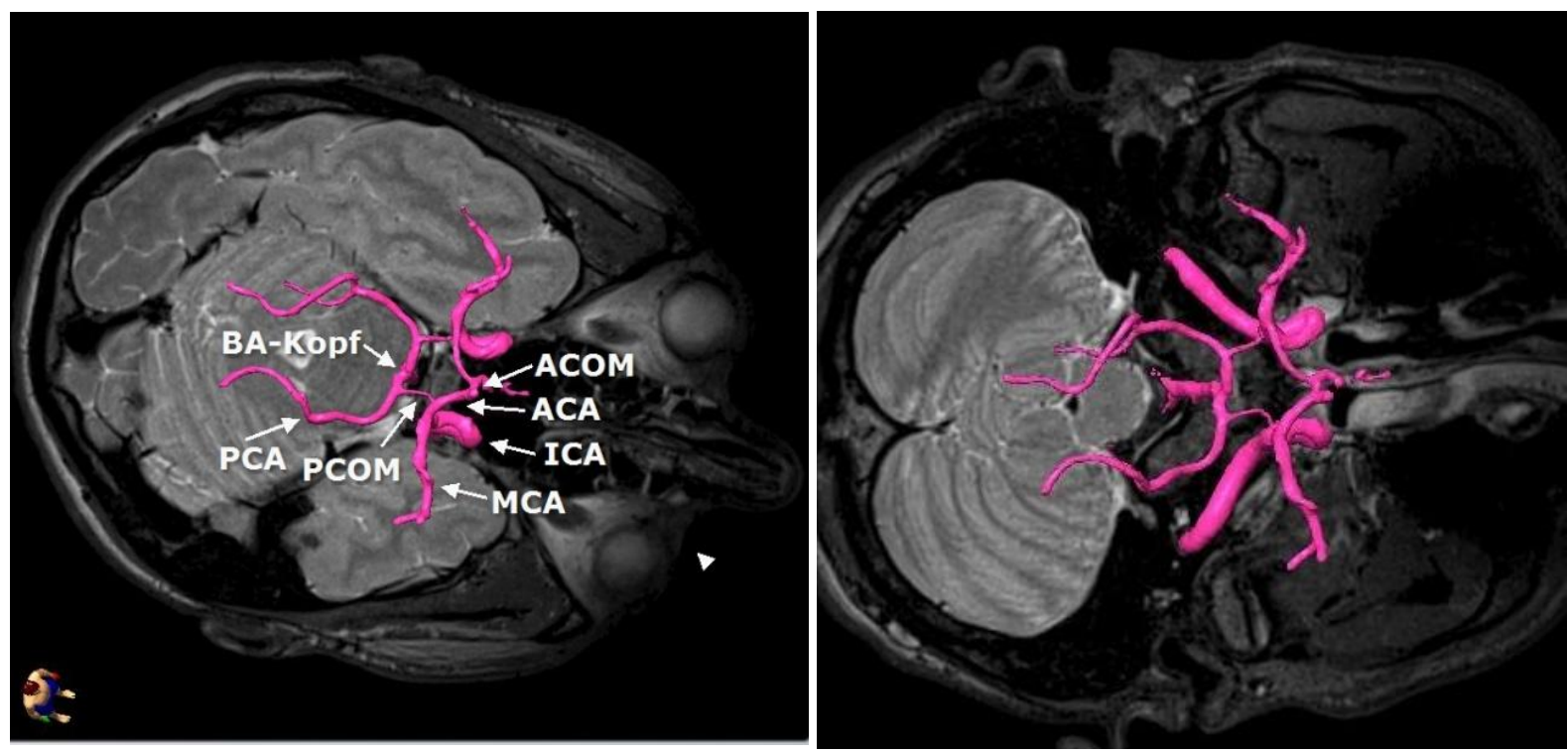

Abb. 2: Normvariante der Hirnbasisarterien dargestellt in einer navigierbaren $3 D$ Rekonstruktion einer Time-of-flight-MRT-Sequenz. A: Ansicht von kranial, B: Ansicht von okzipito-kranial.

\section{Gefäßidentifikation}

Allgemeine Kriterien, die zur Zuordnung eines Dopplersignals zu einem bestimmten intrakraniellen Gefäßsegment herangezogen werden (vgl. auch Tab. 2), sind die Fokustiefe des Ultraschalls (also die Messtiefe), die Einschallrichtung, die Strömungsrichtung des Blutes 
(auf die Sonde zu- oder von der Sonde wegfließend), die Strömungsgeschwindigkeit und die Zuhilfenahme des Kompressionstests der Arteria carotis communis (Nicoletto und Burkman, 2009).

\begin{tabular}{|c|c|c|c|c|}
\hline$\underline{\text { Gefäß }}$ & $\underline{\text { Tiefe }(\mathbf{m m})}$ & $\underline{\text { Strömungsrichtung }}$ & $\underline{\text { ipsilat. Carotiskompression }}$ & $\underline{\text { kontralat. Carotiskompression }}$ \\
\hline MCA & $35-55$ & Zur Sonde & Strömungsabnahme & Kein Effekt \\
\hline ACA & $70-75$ & Von der Sonde weg & Meist Strömungsumkehr & Leichte Strömungszunahme \\
\hline PCA & $65-75$ & Zur Sonde hin & Leichte Strömungszunahme & Kein Effekt \\
\hline ICA & $60-65$ & Zur Sonde hin & Meist Nullfluss & Evtl. leichte Strömungszunahme \\
\hline
\end{tabular}

Tab. 2: TCD - Identifizierungskriterien der basalen Hirnarterien (nach Widder und Görtler 2004)

Aufgrund der engen topographischen Beziehungen und der zahlreichen anatomischen Varianten sind eindeutige Zuweisungen aber nicht immer sicher möglich.

\section{Arteria cerebri media (MCA)}

Bei transtemporaler Beschallung kann in einer Fokustiefe von ca. $50 \mathrm{~mm}$ ein anterogrades Flusssignal der MCA erwartet werden, das in die Tiefe bis zur Karotisbifurkation verfolgbar ist (bidirektionales Flussbild mit Anteilen der ACA). Im Verlauf des Gefäßes nach peripher können Mischsignale auftreten, die durch Abgänge aus dem Hauptstamm der MCA verursacht werden. In der Mehrzahl der Fälle verzweigt sich die MCA in der Insel in ihre Äste. In 8,5\% der Fälle teilt sie sich allerdings bereits direkt nach dem Abgang aus A. carotis interna (Widder 1987). Eine Kompression der A. carotis communis führt zu einer Flussabnahme in der MCA (Diehl und Berlit 1996). Da in dieser Messtiefe kein anderes Signal zu erwarten ist, sind Verwechselungen mit anderen Gefäßen selten. Aus eigener 
Erfahrung besteht aber in Messtiefen ab $50 \mathrm{~mm}$ potenziell die Gefahr der Verwechslung mit dem P1-Segment der PCA (siehe S. 34, Fallillustration in Kap. 4.1).

\section{Arteria cerebri anterior (ACA)}

Die ACA findet sich meist in einer Tiefe von 65-75 mm, wobei das Flusssignal von der Sonde weg zeigt. Nach lateral ist das Signal, wie schon oben beschrieben, ebenfalls bis zur Karotisbifurkation verfolgbar. Aufgrund des Gefäßverlaufs in Richtung kranial ist das A2Segment häufig nicht gut beurteilbar, da der Insonationswinkel nahezu senkrecht werden kann, mangels der Kenntnis über die individuelle Anatomie jedoch keine sinnvolle Winkelkorrektur möglich ist. Im Bereich der Mittellinie werden häufig Mischsignale abgeleitet, da ipsilaterale und kontralaterale ACA hier sehr nahe beieinander liegen. Die Kompression der ipsilateralen ACC sollte eine Flussumkehr in der ACA zur Folge haben. Wird die kontralaterale ACC komprimiert, so kann es zu einer Flusserhöhung in der ACA kommen.

\section{Arteria cerebri posterior (PCA)}

In einer Beschallungstiefe von $75 \mathrm{~mm}$ kann meist der Kopf der Arteria basilaris abgeleitet werden. Hier teilt sich die BA in die beiden PCA. Aus diesem Grund kann hier ein bidirektionaler Fluss erwartet werden, der nach peripher verfolgbar ist. Im nichtpathologischen Fall sind die Flussgeschwindigkeiten niedriger als in der MCA. Häufig kehrt sich im P2-Segment (nach dem PCOM-Ast) die Flussrichtung um, da die PCA hier nach okzipital umschlägt. In einer Publikation, für die 1000 Präparate untersucht wurden, wird in 10,6\% der Fälle von hypoplastischen P1-Segmenten berichtet (Kapoor et al. 2008). Da in diesem Fall die PCA nicht bis zum Basilariskopf verfolgbar sein kann, besteht die Gefahr der Verwechslung mit der MCA (Jatuzis et al. 2000). Bei ipsilateraler Karotiskompression kann 
im P1-Segment eine leichte Strömungszunahme erwartet werden, da über die PCOM Blut in das ICA-Stromgebiet geleitet werden kann.

\section{Arteria carotis interna (ICA)}

Ausgehend vom Hauptstamm der MCA kann, wie oben beschrieben, das Karotis-T aufgesucht werden (meist in einer Tiefe von ca. $60 \mathrm{~mm}$ ). Kaudal davon wird die Endstrecke der ICA im Karotissiphon von transtemporal beschallt. Aufgrund der charakteristischen Siphon-Anatomie sind sowohl orthograde als auch retrograde Flüsse ableitbar, die im oberen Gefäßsegment zumeist orthograd sind. Da in dieser Untersuchungstiefe aber auch Signale der PCA und ACA erwartet werden können, ist eine Differenzierung oft nur durch einen Kompressionstest möglich. Komprimiert man die ipsilaterale ACC, so sollte die Flussgeschwindigkeit in der ICA abnehmen; in der PCA wäre eventuell eine leichte Flusszunahme möglich. Bei funktionell offener ACOM kann sich die Flussrichtung in der ACA umkehren.

\section{Arteria vertebralis (VA) und Arteria basilaris $(B A)$}

Die Arteria basilaris kann gewöhnlich in ihrem Endabschnitt (Basilaris-Kopf) über das transtemporale Schallfenster insoniert werden. Eine Ableitung des Hauptstammes und der Vertebralarterien ist hingegen nur über eine transforaminale Beschallung möglich. Da die Dicke der Weichteile am kraniozervikalen Übergang allerdings erhebliche individuelle Unterschiede aufweist (Lang 1979), variiert die Insonationstiefe, in der die VA und die BA erwartet werden können, erheblich (45-70 mm für VA, 80-110 mm für BA). Aufgrund der engen anatomischen Nachbarschaft der Vertebralarterien ist eine Differenzierung bei gleicher Flussgeschwindigkeit nur über Kompression der Atlasschlingen möglich. Auch die Unterscheidung zwischen Endabschnitt der VA und Anfangssegment der BA ist schwierig, da 
kein anatomischer Orientierungspunkt existiert und beide Gefäße gleichsinnige Flussrichtungen aufweisen. Die sichere Differenzierung gelingt auch hier nur über den Kompressionstest, auf dessen Schwierigkeiten und Risiken später in diesem Kapitel eingegangen werden soll.

\section{Varianten des Circulus arteriosus Willisi}

Der Circulus arteriosus Willisi besteht aus ACA, ACOM, ICA, PCA und PCOM. Durch die Kommunikation des vorderen und des hinteren Stromgebiets über ACOM und PCOM besteht die Möglichkeit, bei Stenosen oder Okklusionen, Kollateralen (dadurch Flussumkehr in einzelnen Segmenten) auszubilden. Aufgrund der Existenz zahlreicher Varianten des Circulus ist eine wie oben beschriebene Zuordnung von Flusssignal zum Gefäß allerdings nicht immer sicher möglich.

In der Literatur wird eine Häufigkeit von $13 \%$ bis $72,8 \%$ für das Vorhandensein der Normvariante des Circulus Willisi berichtet. In einer Studie von Kapoor et al. wurde die Gefäßanatomie an 1000 menschlichen Gehirnen untersucht. Hierbei zeigte sich, dass in 54,8\% der Fälle eine Abweichung von der Norm (= Fehlen, Multiplikation oder Hypoplasie einer Arterie) besteht (Kapoor et al. 2008). Ähnliche Ergebnisse zeigte die Arbeit von KrabbeHartkamp et al. (Krabbe-Hartkamp et al. 1998).

Die Differenzierung der Ergebnisse von Kapoor et al. ist in der folgenden Tabelle ersichtlich: 


\begin{tabular}{|c|c|c|c|c|}
\hline Gefäß & Fehlen & Hypoplasie & Multiplikation & Summe \\
\hline ACA & $0,4 \%$ & $1,7 \%$ & $5,8 \%$ & $7,9 \%$ \\
\hline PCA & $0,0 \%$ & $10,6 \%$ & $3,1 \%$ & $13,7 \%$ \\
\hline ACOM & $1,8 \%$ & $2,1 \%$ & $11,6 \%$ & $15,5 \%$ \\
\hline PCOM & $1,0 \%$ & $16,7 \%$ & $0,0 \%$ & $17,7 \%$ \\
\hline Gesamt & $\mathbf{3 , 2} \%$ & $\mathbf{3 1 , 1} \%$ & $\mathbf{2 0 , 5} \%$ & $\mathbf{5 4 , 8} \%$ \\
\hline
\end{tabular}

Tab. 3: Varianten des Circulus Willisi (nach Kapoor et al. 2008)

\section{Kompressionstests als Hilfsmittel}

Der Karotis-Kompressionstest ist ein wichtiges, aber invasives, Hilfsmittel zur Identifikation von Gefäßen während der konventionellen TCD-Untersuchung. Durch Abdrücken der Blutversorgung auf einer Seite des vorderen Stromgebietes kann es zu Strömungsveränderungen (siehe S. 10, Tab. 2) in einzelnen Segmenten kommen, da vermehrt Blut über die Kollateralen ACOM und PCOM fließt.

Das Ergebnis ist allerdings abhängig von der Vollständigkeit des Circulus Willisi und somit auch von der Möglichkeit, Kollateralkreisläufe auszubilden. Da aber entweder ACA, PCA, ACOM oder PCOM in insgesamt 34,2\% der Fälle nicht vorhanden oder hypoplastisch sind (Kapoor et al. 2008), kann der Test nicht immer als sicheres Hilfsmittel herangezogen werden. Ferner ist der Test umstritten, weil Fallberichte beschreiben, dass durch Kompression der Karotiden Emboli aus Plaques gestreut wurden, die zu neurologischen Defiziten führten (Khaffaf et al. 1994).

Nicht so sicher und weniger konstant wie die Kompression der ACC gelingt der Kompressionstest der A. vertebralis an der Atlasschlinge. Eine Schwierigkeit ist die durch die 
Kompression der Gefäße verursachte Verschiebung der Weichteile, die häufig mit einem Signalverlust der TCD vergesellschaftet ist (von Reutern und Büdingen 1993).

\subsubsection{Winkelkorrektur}

Weil es sich bei der transkraniellen Dopplersonographie nicht um ein schnittbildgebendes Verfahren handelt, kann während der Untersuchung kein sicherer Rückschluss darauf gezogen werden, in welchem Winkel ein Gefäß insoniert wird. Wahre Messwerte ergeben sich in der Dopplersonographie allerdings nur dann, wenn die Achse des Schallstrahls in der Strömungsachse des Blutgefäßes liegt (wenn $\alpha=0^{\circ}$, dann $\cos \alpha=1$ ) (Widder 1987). Da dies aufgrund der natürlichen Gefäßanatomie aber nur selten der Fall ist, werden mit der TCD signifikant zu geringe Blutflussgeschwindigkeiten gemessen (Martin et al. 1995). Die wahre Strömungsgeschwindigkeit ergibt sich mathematisch durch eine Winkelkorrektur, die die Kenntnis des Einstrahlwinkels erfordert (von Reutern und Büdingen 1993):

$\mathrm{v}=\Delta \mathrm{f} * \mathrm{c} / 2 * \mathrm{f} * \cos \alpha \quad(\mathrm{v}=$ Geschwindigkeit; $\mathrm{f}=$ Frequenz; $\mathrm{c}=$

Ausbreitungsgeschwindigkeit, $\alpha=$ Einschallwinkel)

Je kleiner der Winkel $\alpha$ ist, desto geringer bleibt auch der Messfehler (vgl. Abb. 4). Trifft der Schall im Winkel von $90^{\circ}$ auf die fließenden Korpuskeln, so ist theoretisch kein Fluss mehr messbar $(\cos 90=0)$. 

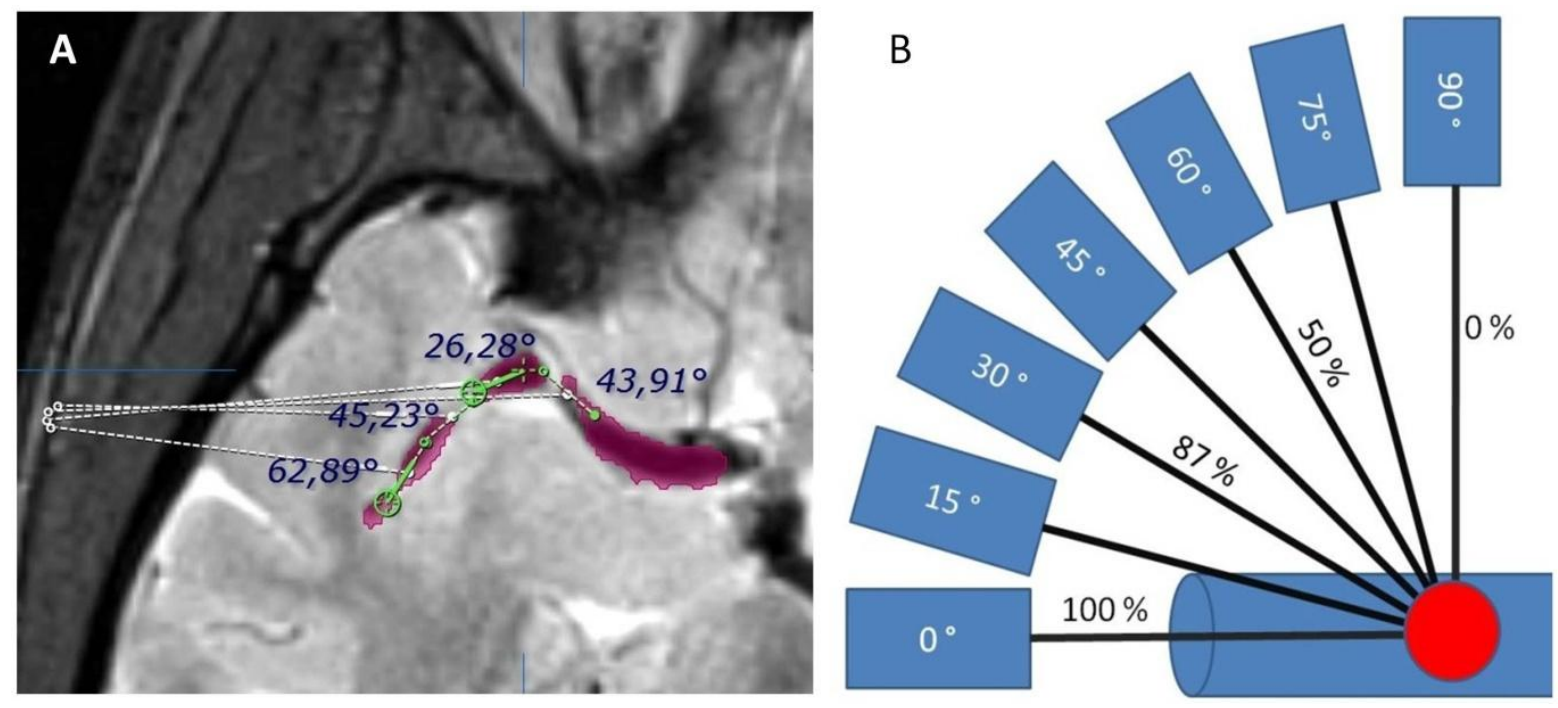

Abb. 4: A: Verlauf der MCA im axialen Schnittbild mit Einschallwinkeln an verschiedenen

Gefäßsegmenten. B: Einfluss des Einstrahlwinkels auf das Messergebnis.

\subsubsection{Transkranielle Duplexsonographie}

Im Gegensatz zur transkraniellen Dopplersonographie ist die transkranielle Duplexsonographie (TCCD), die in den letzten Jahren vermehrt Einzug in die Klinik gehalten hat, eine B-Bild-gebende Methode, die zwei wichtige Vorteile gegenüber der TCD aufweist. Zum einen wird anhand von Schnittbildern die Identifizierung des Gefäßes erleichtert, zum anderen ermöglicht die Kenntnis des anatomischen Gefäßverlaufs die Winkelkorrektur der Flussgeschwindigkeiten in den beschallten Blutgefäßen (Martin et al. 1995).

Ein limitierender Faktor ist allerdings die Tatsache, dass für die Darstellung des Hirnparenchyms eine hohe Auflösung benötigt wird, die über Schallfrequenzen von 2-3 MHz erzielt wird. In der Literatur wird in 10-20\% der Fälle über ein inadäquates Schallfenster berichtet (Seidel et al. 1995, Gerriets et al. 1999). Da die TCD ohne die bildgebende Auflösung auskommt, ist es prinzipiell möglich, bei schlechten Schallfensterverhältnissen 
eine Sonde mit niedrigeren Frequenzen $\mathrm{zu}$ verwenden, da mit niedrigeren Ultraschallfrequenzen (1 MHz) der Knochen besser durchdrungen werden kann (Georgiadis et al. 1999, Klötzsch et al. 1998). Dabei muss allerdings in Kauf genommen werden, dass somit keine anatomisch bildgebende Schnittbilddiagnostik zur Verfügung steht.

\subsection{Neuronavigation in der Neurochirurgie}

Durch Einführung der Neuronavigation in neurochirurgische Operationsverfahren ist es möglich geworden, (auch tiefliegende) Hirnstrukturen operativ sicher und parenchymschonend zu erreichen. Im Folgenden soll kurz auf die allgemeinen Grundlagen und die Anwendung von Navigationsgeräten in der Neurochirurgie eingegangen werden.

Einzug in den klinischen Alltag erhielt die rahmenlose computergestützte Neuronavigation im Jahr 1986. Roberts et al. beschrieben eine Methode, mit deren Hilfe die Integration eines präoperativen CT-Scans in das Echtzeit-Sichtfeld eines Operationsmikroskops gelang (Roberts et al. 1986). Im Gegensatz zu der bis dahin häufig genutzten stereotaktischen Navigation war es nun möglich, die Position eines Operationsinstruments (im Fall von Roberts die Fokustiefe des Mikroskops) in Echtzeit in Korrelation zum Navigationsdatensatz eines präoperativen CT- oder MRT-Scans zu sehen.

\subsubsection{Technische Grundlagen und präoperatives Vorgehen}

Viele der heutzutage am Markt erhältlichen und klinisch verwendeten Neuronavigationssysteme sind funktionell ähnlich (Lumenta 2008). Im Folgenden soll die Funktionsweise dieser Geräte am Beispiel des Kolibri-Systems (Brainlab AG, Feldkirchen, Deutschland, vgl. S. 22, Kap. 3.2) erläutert werden. 
Das Navigationssystem besteht aus folgenden Grundkomponenten: Planungs-Workstation, Navigationscomputer mit Kamerasystem und Infrarotleuchten, Patienten-Reflexmarker-Stern sowie Operations- und Registrierinstrumente. Als Navigationsdatensätze dienen präoperative CT- oder MRT-Schichtbildaufnahmen, die vom Navigationscomputer in triplanare Datensätze rekonstruiert werden. Voraussetzung für die einwandfreie Kompatibilität sind lückenlose axiale Scans mit einer Schichtdicke von maximal $2 \mathrm{~mm}$.

Das vom Navigationsgerät ausgestrahlte Infrarot-Licht wird passiv von den Reflexmarkern des rigide am Patientenkopf befestigten Dreisterns reflektiert. Zwei Kameras registrieren die Position dieser Marker und errechnen nach manueller Registrierung von bis zu fünf auf die Kopfhaut geklebten Oberflächenmarkern (Fiducials) die Position des Kopfes. Die Spitze der ebenfalls mit Reflexmarkern bestückten Registrier- und Operationsinstrumente, deren Geometrie vorkalibriert ist bzw. manuell kalibriert werden kann (Muacevic et al. 2000), kann dann intraoperativ in Relation zum 3D-Bilddatensatz gesehen und als Raumkoordinate erfasst werden (Gumprecht et al. 1999).

Eine manuelle Registrierung (auch Referenzierung genannt) ist notwendig, um eine räumliche Echtzeit-Relation zwischen Schichtbilddatensatz und der Anatomie des Kopfes herzustellen (Grunert et al. 2003). Zur Referenzierung muss die Position des Kopfes in Beziehung zum Reflexmarkerstern registriert werden. Hierzu werden dem Patienten vor der präoperativen MRT- oder CT-Bildgebung in der Regel Oberflächenmarker mit Kontrastmittelkugeln auf die Kopfhaut geklebt, deren Positionen anschließend zuerst an der Planungs-Workstation im Schichtbilddatensatz bestimmt und später am Navigationssystem mittels eines vorkalibrierten Pointers manuell am Kopf registriert werden (= Fiducial-based-paired-point-Transformation). Alternativ kann die Hautoberfläche mittels eines Instruments, das die Hautimpedanz misst (Soft-Touch $®)$, oder eines Laserinstruments (Z-Touch®) abgetastet werden und somit über 
Softwarealgorithmen eine Patientenregistrierung erfolgen (=Surface-contour-matching) (Akamatsu et al. 2009, Grunert et al. 2003).

\subsubsection{Genauigkeit der Neuronavigation}

In der Literatur existieren viele wissenschaftliche Arbeiten über die Genauigkeit von Neuronavigationsverfahren, die sich jedoch in den weitaus meisten Fällen auf spezielle Anwendungsgebiete beziehen. Grunert et al. beschäftigten sich 2003 in einem Review mit der allgemeinen Navigationsgenauigkeit. Dabei unterscheidet er Technik-, Registrier- und Anwenderfehler als Ursachen für mögliche Ungenauigkeit. Technisch bedingte Fehler seien am wenigsten bedeutend $(0,2-0,6 \mathrm{~mm})$. Die Ungenauigkeit aufgrund eines Registrierfehlers sei hingegen mit 0,2 - 3,0 mm größer und mögliche Anwenderfehler seien schwierig abzuschätzen. Dieser betrage in Abhängigkeit von Zeit, Ort der Operation und Liquor-Lecks 0,6 - 10,0 mm (Grunert et al. 2003).

\subsubsection{Klinische Anwendung in der Neurochirurgie}

Grundsätzlich kann die Neuronavigation als anatomische Orientierungshilfe bei jeder Art von neurochirurgischen Operationen dienen. In den vergangenen Jahrzehnten hat sich das Verfahren etabliert und geholfen, neurochirurgische Eingriffe gezielter auf die Pathologie abzustimmen (Maciunas 2006), da mithilfe einer genauen Vektorplanung der operative Zugangsweg klein gehalten und die Pathologie auf direktem Wege angegangen werden kann. Weil mit diesem Verfahren aber auch zusätzliche Kosten und weiterer Aufwand, wie z.B. das Anfertigen und die Planung von Navigationsdatensätzen (Gumprecht et al. 1999), verbunden sind, wurde es lange von vielen Operateuren nicht regelhaft verwendet. Etabliert hat sich die Anwendung allerdings bei folgenden Eingriffen: Lokalisation sowie Zugangs- und Kraniotomieplanung von intrakraniellen Tumoren, Schädelbasischirurgie, intrazerebrale 
Biopsien, Endoskopien und Katheterplatzierungen in der funktionellen Neurochirurgie (Gumprecht et al. 1999). Noch weniger verbreitet ist die Neuronavigation in der Wirbelsäulenchirurgie. Auch die intraoperative Integration der intrazerebralen BBildsonographie hat sich in den vergangenen Jahren etabliert (Akdemir et al. 2007, Mathiesen et al. 2007).

\subsubsection{Autopilot-Funktion}

In vielen Anwendungsbereichen von Neuronavigationssystemen (z.B. Biopsie-Entnahme) kann die sogenannte „Autopilot-Funktion“ zur Hilfe herangezogen werden. Diese liefert zusätzlich zur triplanaren Schichtbildrekonstruktion eine visuelle Information über Eindringtiefe und Winkelkongruenz des Operationsinstruments in Bezug zu einer Navigationstrajektorie (Ringel et al. 2009). Eine Navigationstrajektorie wird durch einen Eintrittspunkt sowie einen Zielpunkt als Strecke im Navigationsdatensatz definiert. Bei der Entnahme einer Tumorbiopsie entspräche der Startpunkt dem Ort des geplanten MiniBohrlochs und der Zielpunkt dem Ort des geplanten Biopsie-Gewebes.

Der Autopilot liefert dem Anwender somit eine einfache optische Orientierungshilfe zur korrekten Haltungsachse seines Instruments (vgl. S. 38, Abb. 15, A). 


\section{Patienten, Material und Methoden}

Es wurden insgesamt 33 nichtinvasiv neuronavigierte transkranielle Doppleruntersuchungen an intensivmedizinisch betreuten Patienten zur Evaluierung der Genauigkeit und klinischen Anwendbarkeit durchgeführt.

Die ethische Vertretbarkeit wurde vorab von der Ethikkommission der Medizinischen Fakultät der Georg August Universität Göttingen unter der Antragsnummer 20/7/10 geprüft und für unbedenklich erklärt.

\subsection{Patientenkollektiv}

An zwölf Patienten, die unabhängig von dieser wissenschaftlichen Studie in der Klinik für Neurochirurgie der Universitätsmedizin Göttingen behandelt wurden, wurden insgesamt 33 neuronavigierte transkranielle Doppleruntersuchungen durchgeführt. Neun der zwölf Patienten waren weiblich, drei männlich. Das mittlere Alter der Patienten betrug 56 Jahre ( \pm 12,7 Jahre). Grund der Hospitalisierung war bei elf der zwölf Patienten eine vorausgegangene subarachnoidale Hirnblutung. Eine Patientin litt unter einer zerebralen Vaskulitis mit konsekutiver intrazerebraler Stammganglienblutung.

Indikationsgerecht wurde bei allen Patienten infolge ihrer Grunderkrankung eine diagnostische computertomographische Angiographie angefertigt, die unabhängig vom wissenschaftlichen Charakter dieser Arbeit war und als Datensatz für die späteren nichtinvasiv neuronavigierten TCD-Untersuchungen geeignet war. Patienten, bei denen kein adäquates Schallfenster auffindbar war, sollten nicht in die Auswertung einbezogen werden. Allerdings konnte mithilfe der Navigation bei allen Patienten ein Schallfenster ausfindig gemacht werden. 


\subsection{Material}

Die Durchführung einer nichtinvasiv neuronavigierten TCD erfordert folgende Materialien: Ein Neuronavigationssystem inklusive Navigationszubehör und Planungssoftware, ein Dopplersonographiegerät mit 2-MHz-pulsed-waved Schallsonde, einen Schallsondenadapter zur Arretierung eines Reflexmarkerdreisterns und Schichtbildaufnahmen der Hirngefäße (zum Navigationssystem kompatibel). Mit Ausnahme des Schallsondenadapters sind alle Gerätschaften am freien Markt erhältlich und in vielen neurochirurgischen Kliniken verfügbar.

Für die Untersuchung dieser Fragestellung wurde als Navigationssystem ein handelsüblicher Brainlab Kolibri ${ }^{\circledR} \quad$ (Brainlab AG, Feldkirchen, Deutschland) mit zugehöriger Planungssoftware iPlan ${ }^{\circledR}$ Cranial 2.6 (ebenfalls Brainlab AG) verwendet. Als TCD-Gerät diente ein DWL MultiDop T digital ${ }^{\circledR}$ (DWL Compumedics Germany GmbH, Singen, Deutschland). Der Kolibri ermöglicht es dem Anwender, nicht vorkalibrierte Instrumente manuell zu registrieren. Da die 2-MHz Schallsonde des DWL MultiDop T digital® (im folgenden MultiDop genannt) aufgrund ihrer Abmessungen nicht kompatibel zu den Referenzsternen des Kolibri ist, wurde von den wissenschaftlichen Werkstätten der Universitätsmedizin Göttingen ein passgenauer, achsengerechter Aufnahmeadapter angefertigt, der die Arretierung eines Reflexmarker-Dreisterns erlaubt (siehe Abb. 5, Mitte) 


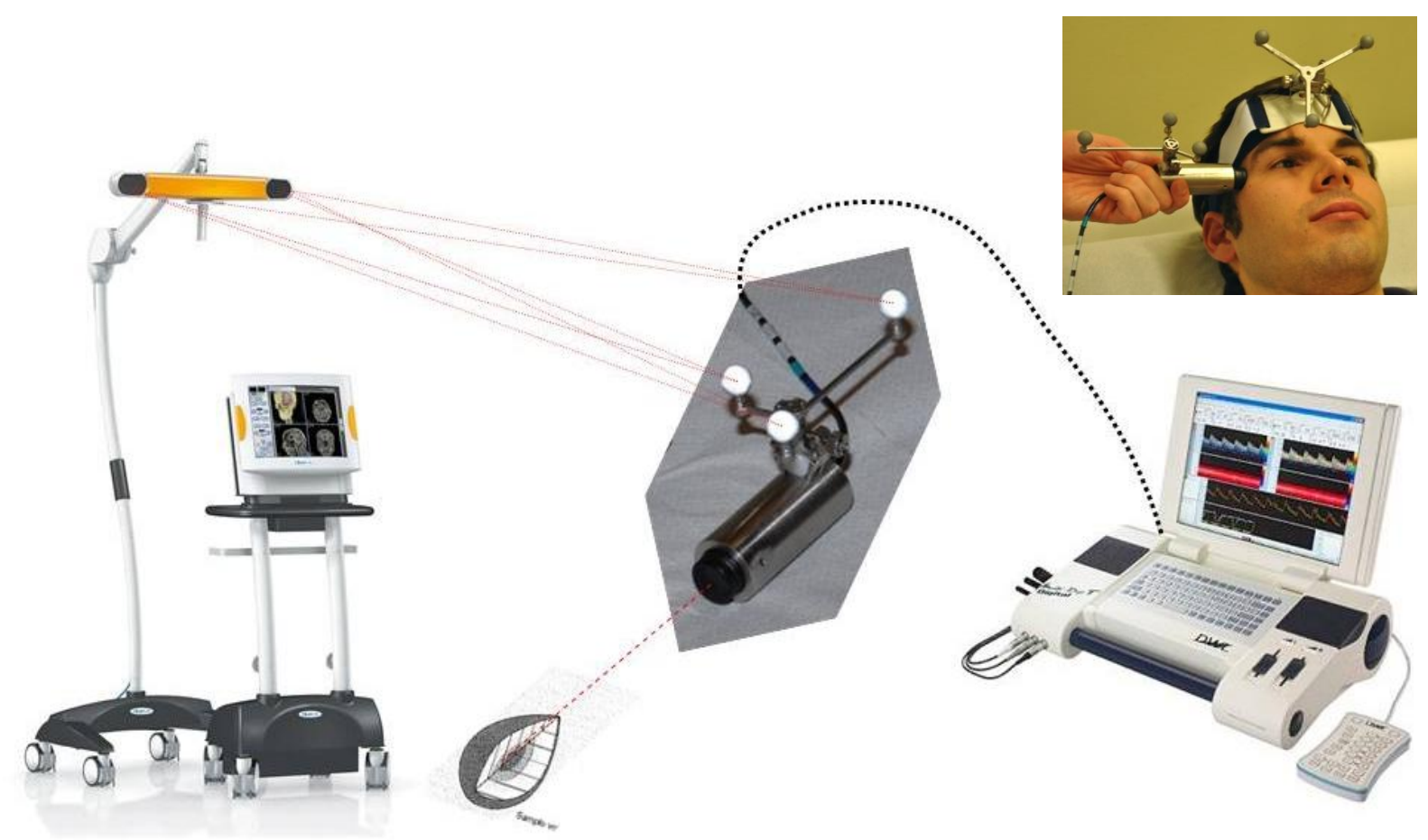

Abb. 5: links: Brainlab Kolibri-Navigationssystem. Mitte: Sondenadapter mit Referenzstern und 2 MHz-TCD-Schallsonde. Rechts unten: DWL MultiDop T digital ${ }^{\mathrm{TM}}$. Rechts oben: Reference Headband und Sonde

\subsection{Methoden}

Zur Überprüfung der Genauigkeit der navigierten TCD wurden bildmorphologisch definierte Raumkoordinaten mit den Koordinaten des Punktes, an dem tatsächlich ein optimales Flusssignal des zu untersuchenden Gefäßsegments gemessen wurde, verglichen und deren räumliche Distanz ausgewertet (siehe S. 35, Abb. 14). Um die Reproduzierbarkeit und Verlässlichkeit der Methode zu evaluieren, wurden die Koordinaten der Messpunkte aus der ersten Untersuchung mit denen der Folgeuntersuchungen verglichen. Die nichtinvasive Patientenregistrierung wurde mittels eines flexiblen, selbstklebenden Kopfbandes und einer anatomischen Landmarkenregistrierung verwirklicht. Um ein potentielles Verrutschen des Kopfbandes zu detektieren und auszuwerten, wurden die Koordinaten von drei (auf der 
Kopfhaut platzierten) Klebemarkern vor der Untersuchung mit denen nach der Untersuchung verglichen. Diese, unter intensivmedizinischen Bedingungen ermittelten Daten, wurden zur Kontrolle mit Daten einer weiteren Untersuchungsreihe verglichen. In diesen unter optimalen Untersuchungskautelen durchgeführten Kontrolluntersuchungen (vgl. S. 39, Kap. 4.3) wurden unmittelbar nach Akquirierung der drei Punkte die Koordinaten durch Aufsetzen des Softtouch-Pointers re-registriert.

\subsubsection{Untersuchungsvorbereitung}

\section{Datentransfer}

Alle verwendeten CT-A wurden unabhängig von dieser Studie in der Abteilung für Neuroradiologie der UMG erstellt (Somatom Definiton AS CT-Scanner (Siemens, Erlangen, Deutschland)). Da die Neuroradiologie mit der Brainlab-Workstation (iPlan 2.6) vernetzt ist, wurden die Datensätze per Netzwerk transferiert, was alternativ auch per Datenträger (z.B. CD-ROM) möglich ist. Am Ende des Planungsvorgangs wurde der vorgeplante Navigationsdatensatz per USB-Massenspeicher auf den Kolibri überspielt.

\section{Referenzierung}

Aufgrund der Tatsache, dass die CT-A-Aufnahmen gänzlich unabhängig von den TCD Untersuchungen angefertigt wurden, musste zur Referenzierung des Patientenkopfes ein Verfahren ohne Fiducial-Akquirierung gewählt werden. Hierzu kommen prinzipiell die

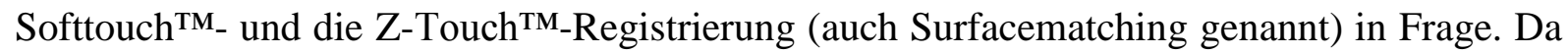
die Scans aber auch zeitlich unabhängig aufgenommen wurden, stellt eine bei intensivmedizinisch behandelten Patienten häufige Schwellung des Gesichts potenziell ein Hindernis zum Surfacematching dar. Um dieses Problem zu vermeiden, wurde in allen 
Untersuchungen eine Landmarkenregistrierung angewendet, die Punkte im Gesicht registriert, die weniger anfällig für ödematöse Veränderungen sind (siehe Abb. 6, links).

Grundlage jeder Landmarkenregistrierung sind anatomische Fixpunkte, die (ähnlich zur Fiducial-Akquirierung) mittels eines vorkalibrierten Pointers zu Beginn jeder Untersuchung registriert werden müssen (Abb. 6, rechts). Als Fixpunkte wurden folgende fünf anatomische Landmarken verwendet, die vor Beginn jeder Untersuchung an der Workstation manuell definiert wurden: Linker lateraler Orbitarand, rechter lateraler Orbitarand, linker Tragus, rechter Tragus und Nasionmitte.

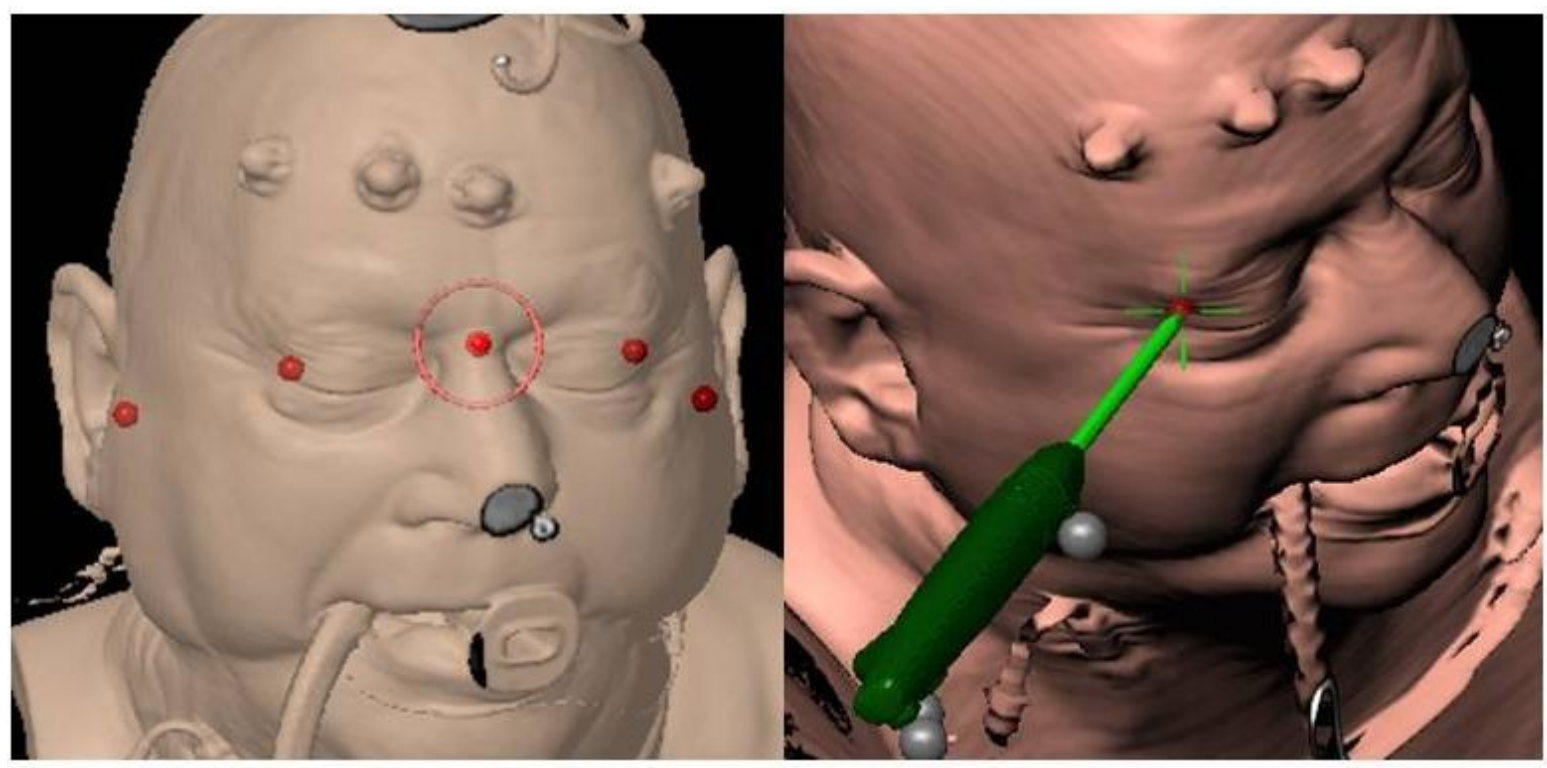

Abb. 6: 3D-Oberflächen-Rekonstruktion eines CT-Datensatzes. Links: anatomische Landmarken, rechts: Registrierung der Landmarken mit einem Softtouch-Pointer.

\section{Gefäßsegmentierung}

Zur einfacheren drei-dimensionalen Orientierung im Gefäßbaum wurden vor jeder Untersuchung die Hirnbasisarterien segmentiert und farblich kodiert. Später konnte der 3DGefäßbaum in einer Übersicht vom übrigen Hirnparenchym freigestellt und aus beliebiger räumlicher Richtung betrachtet werden (vgl. Abb. 7, oben). 


\section{Definition der Gefäßtargets}

Unter der Annahme, dass im Zentrum des Gefäßlumens ein optimales Flusssignal messbar sei, wurden, ebenfalls prä-prozedural, je Untersuchung drei Gefäßtargets gesetzt (vgl. Abb. 7):

1.) M1 Segment MCA,

2.) Karotis $\mathrm{T}$ (= Abgang MCA/ACA aus der ICA)

3.) BA-Kopf (= Abgang der PCA aus BA).

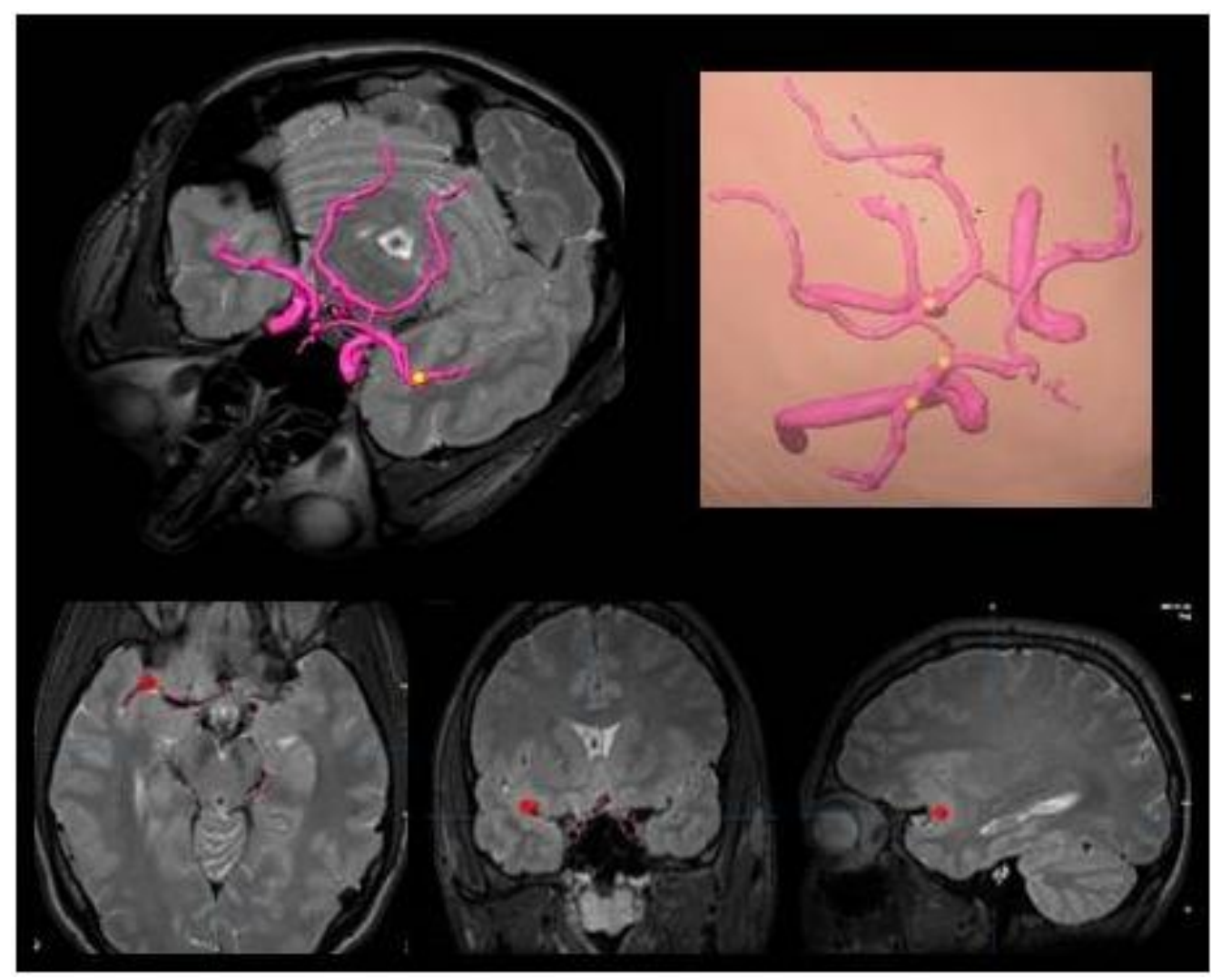

Abb. 7: Gefäßtargets in der MCA, Karotis-T und BA-Kopf. Oben: 3D-Gefäßbaum. Unten: triplanare MRT-Schichtbildgebung.

\section{Patientenreferenzierung}

Mittels eines als Zubehör erhältlichen flexiblen Kopfbandes (Reference Headband, Brainlab), welches durch ein spezielles doppelseitig klebendes Band und einem Klettverschluss fixiert 
wird, entsteht eine ausreichend rigide Verbindung zwischen Reflexmarker-Stern und Patientenkopf (siehe S.23, Abb. 5). Die Referenzierung der Landmarken ermöglicht dem Navigationsrechner eine räumliche Zuordnung von Schichtbildaufnahmen zum Patientenkopf. Nach Referenzierung und vor Beginn der TCD wurde vom Untersucher eine Plausibilitätsprüfung durchgeführt, indem die Spitze des Pointers auf die Mitte des Nasions und die beidseitigen Tragi gehalten wurde und somit die Kongruenz zwischen Bildmorphologie und realer Anatomie überprüft wurde. Diese anatomische Übereinstimmung war Voraussetzung für den Beginn jeder navigierten TCD-Untersuchung.

\section{Schallsonden-Registrierung}

Als letzter Schritt vor Beginn jeder Untersuchung musste die Ultraschallsonde als Instrument am Navigationssystem kalibriert werden. Wie oben schon erwähnt, ist aufgrund der Abmessungen der Sonde kein passender Referenzstern als Zubehör erhältlich. Aus diesem Grund wurde die Sonde in einem speziell angefertigten Aufnahmeadapter mithilfe eines Referenzierungsblocks kalibriert.

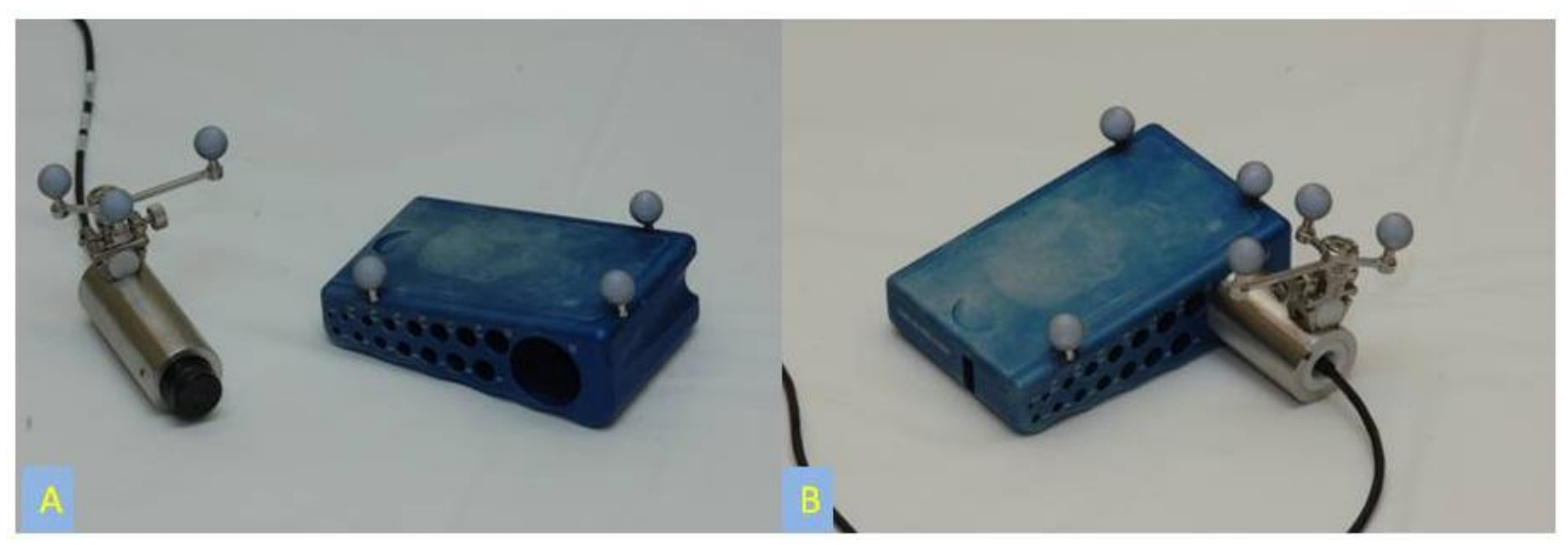

Abb. 8: A: Ultraschallsonde und Referenzierungsblock, B: Sonde im Referenzierungsblock 
Die virtuelle Instrumentenverlängerung, deren Achse die Ultraschallachse darstellt, kann beliebig in $1 \mathrm{~mm}$-Schritten definiert werden und wurde während der Untersuchung der Messtiefe des Ultraschallgeräts angepasst. Der Durchmesser der virtuellen Verlängerung wurde, dem Durchmesser des Messvolumens (Sample Volume) entsprechend, auf 3mm gleichgesetzt.

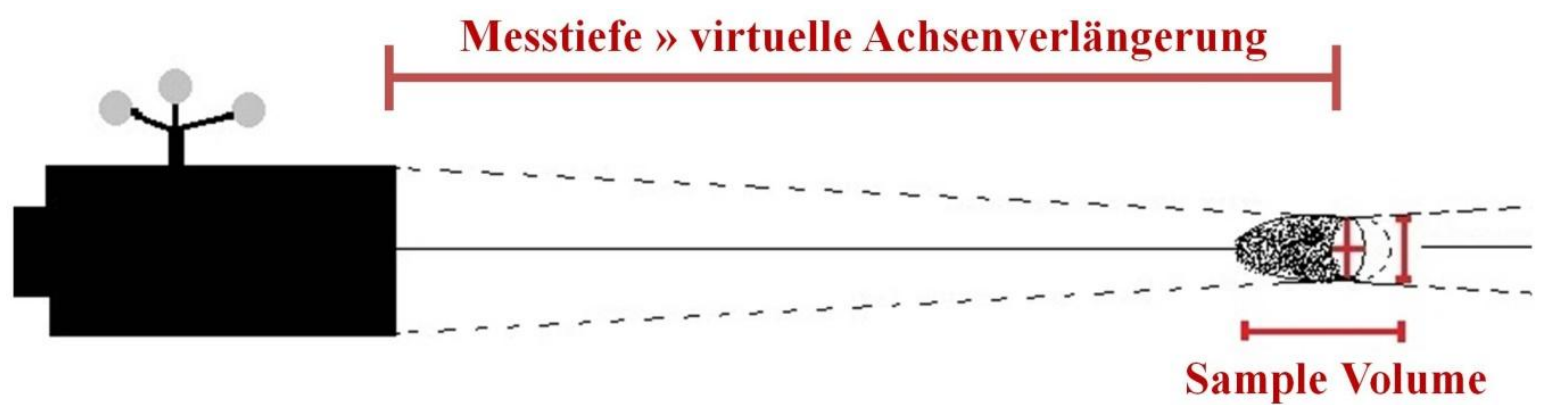

Abb. 9: Skizze der Ultraschallsonde. Die Messtiefe des Messvolumens (=Sample Volume) ist am MultiDop einstellbar. Die virtuelle Achsenverlängerung wird am Navigationsgerät manuell der Messtiefe angepasst.

\subsubsection{Untersuchung / Messung}

Zu Beginn der Messungen wurden bei den Patienten drei Klebemarker auf der Kopfhaut angebracht, deren Koordinaten durch Aufsetzen der Pointerspitze akquiriert wurden. Diese Punkte waren unabhängig zum Kopfband, was ein mögliches Verrutschen des Kopfbandes durch Änderung der Koordinaten der Klebemarker auffällig machte.

Wie unter 3.3.1 beschrieben, wurden je Untersuchung drei Gefäßtargets im Zentrum des Gefäßlumens definiert. Nachdem unter Zuhilfenahme des Doppler-M-Modes ein geeignetes transtemporales Schallfenster ausfindig gemacht wurde, wurden diese Punkte navigiert detektiert und das korrespondierende Flusssignal dokumentiert. Daran anschließend wurde durch manuelle Korrektur der Sondenposition das beste messbare Flusssignal gesucht (vgl. S. 35, Abb. 14) und die zugehörigen Navigationskoordinaten dieses Signals als Trajektorie 
akquiriert, wodurch ein wiederholtes Detektieren dieses Messpunktes mit dem Autopiloten ermöglicht wurde. Die Achse dieser Trajektorie läuft dabei durch das Ultraschallfenster und das intrazerebrale Gefäßtarget. Die Distanz zwischen Spitze der Doppler-Sonde und Koordinate des Targets definiert die Messtiefe des Ultraschalls.

Die Software des MultiDop gibt die Qualität des Messsignals anhand einer Farbskala aus, die eine Aussage über die Intensität des reflektierten Ultraschalls macht. Diese Skala wurde zur Beurteilung des Signals herangezogen (vgl. Abb. 10).

In mehreren seriellen Untersuchungsfolgen wurden die Messungen an denselben Targets und Trajektorien wiederholt. Der Autopilot gibt dem Anwender optische Informationen zur korrekten Positionierung des Instruments (vgl. S. 20, Kap. 2.3.4), die durch die Trajektorie vorgegeben war. In diesem Fall diente er dazu, die Doppler-Sonde in der richtigen Achse auszurichten und die richtige Untersuchungstiefe zu wählen.

Vor Beendigung jeder 3D-Navigation wurden die Koordinaten der Klebemarker überprüft und die Distanz zum eingangs akquirierten Punkt gemessen. 


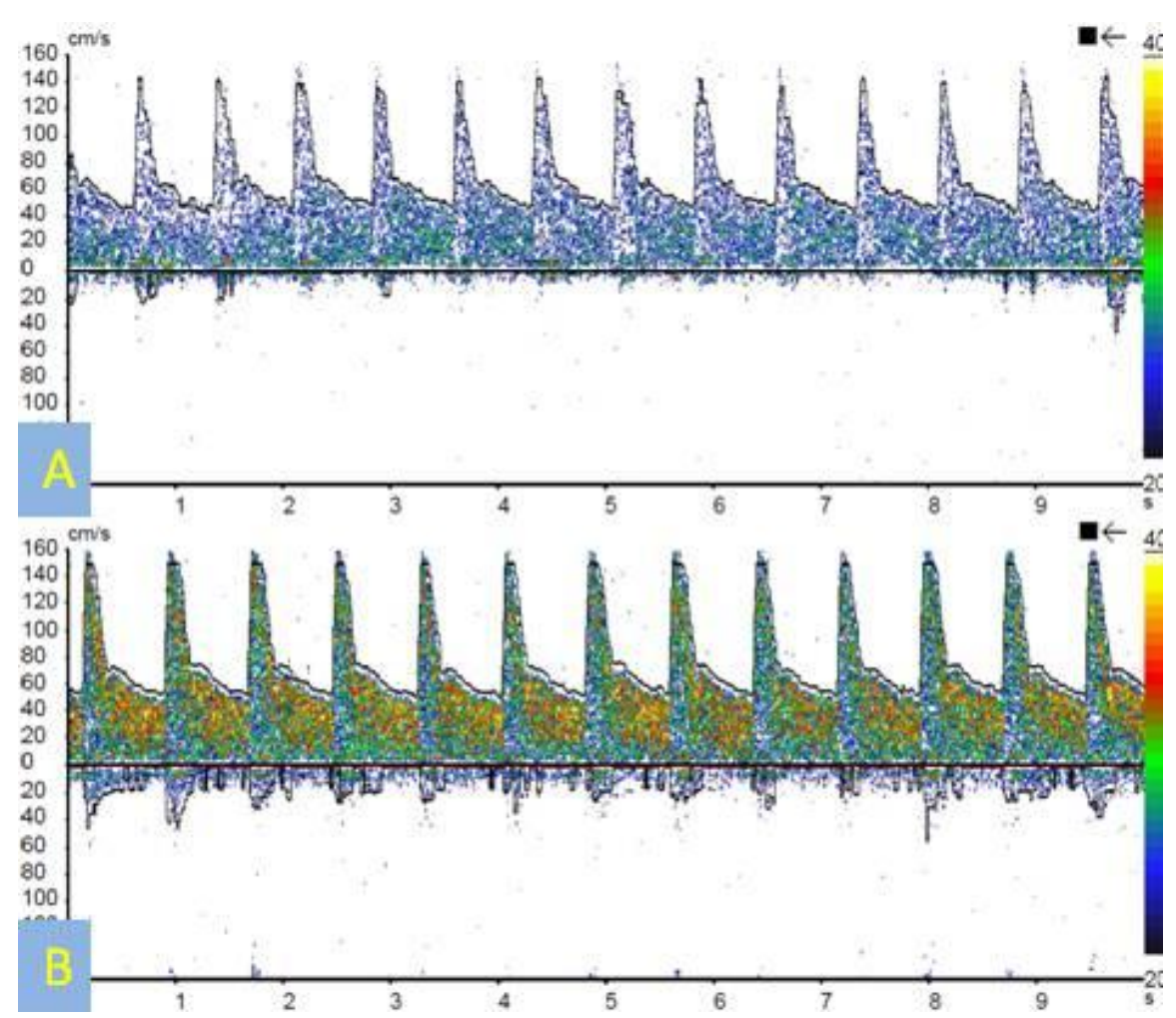

Abb. 10: Flusssignale unterschiedlicher Qualitäten. A: Signal am Gefäßtarget. B: durch Nachjustierung der Sonde wurde das beste messbare Signal gesucht. Die Qualität des Ultraschallsignals wird anhand einer Farbscala am rechten Bildrand dargestellt.

\subsubsection{Datenauswertung / Statistik}

Alle Koordinaten der Gefäßtargets und der Trajektorien der Messpunkte werden vom KolibriSystem in einer Datei in Form von x-y-z-Koordinaten, deren Referenzpunkt die Mitte des Reflexmarker-Dreistern ist, gespeichert. Ein vom Hersteller speziell entwickeltes und für die Durchführung der Studie zur Verfügung gestelltes Software-Tool erlaubt die Auswertung von Distanzen zwischen diesen Punkten. Durch Aufsetzten des Pointers auf die Klebemarker wurden dem Untersucher im Display des Kolibri Distanzen zu den anfangs akquirierten Punkten angezeigt (Millimeter-Einheit mit einer Dezimalstelle) und manuell durch Screenshots dokumentiert. 
Die Daten wurden als Normalwerte mit Mittelwert und Standardabweichung (gerundet auf 2 Dezimalstellen) angegeben. Der unverbundene „Student's t-test““ wurde zur statistischen Signifikanzprüfung (siehe S. 23. Kapitel 3.3) verwendet; $p<0,05$ wurde dabei als signifikant gewertet. 


\section{Ergebnisse}

\subsection{Identifikation von spezifischen Gefäßsegmenten}

Das Prinzip der navigierten TCD besteht darin, dass der Ultraschallfokus gezielt auf ein Gefäßsegment gerichtet werden kann (Abb. 11). Die folgenden Ergebnisse zeigen, wie häufig ein korrespondierendes Flusssignal abgeleitet werden konnte, wenn die TCD-Sonde allein mit Hilfe der Neuronavigation auf das Gefäß gerichtet wurde und erst daran anschließend das TCD-Gerät aktiviert wurde.

Nach Aufsuchen eines adäquaten Ultraschallfensters, gelang die allein neuronavigierte Detektion eines zugehörigen Flusssignals zum vordefinierten Gefäßtarget für die MCA in allen Fällen (n=29). Das Flusssignal des Karotis-T wurde in $81 \%(n=27)$ und das der Basilarisspitze in $90 \%(n=29)$ der Fälle abgeleitet.

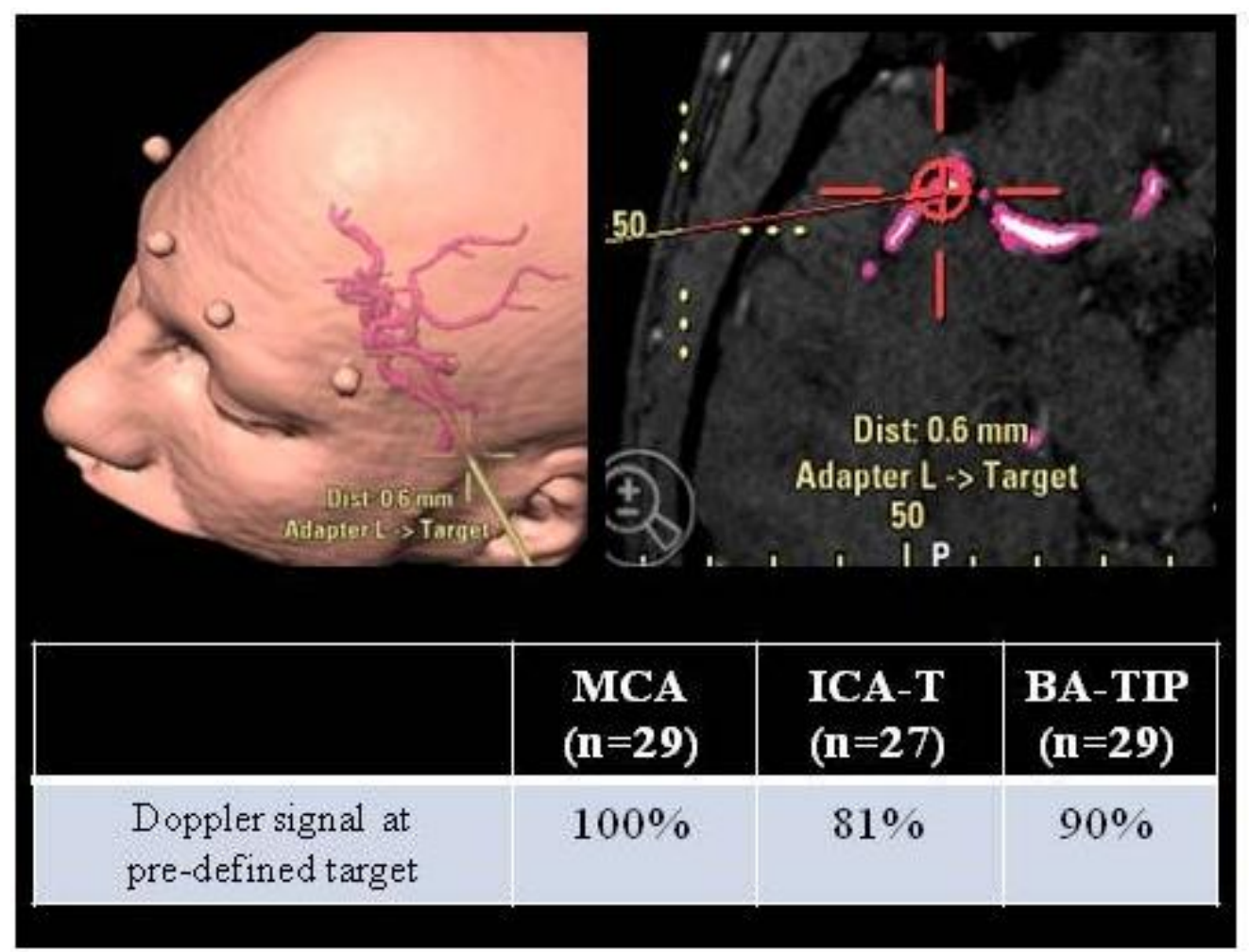

Abb. 11: Detektion eines Gefäßsegments allein durch Neuronavigation 


\section{Fallillustration Gefäßidentifikation}

Wie in Kapitel 2.2.2.2 beschrieben, liegt eine wesentliche Schwierigkeit bei TCDUntersuchungen in der sicheren Gefäßidentifikation. Die Erweiterung der TCD um eine Bildführung kann helfen, diese potentielle Fehlerquelle zu reduzieren. Die folgende Fallillustration zeigt, dass die Zuordnung von Dopplersignalen $\mathrm{zu}$ anatomischen Gefäßsegmenten durch die Neuronavigation erleichtert werden kann.

Während des konventionellen Vasospasmusmonitorings nach SAB fiel bei den täglichen Kontrollen auf, dass die Messwerte trotz identischer Messtiefe erheblichen Schwankungen unterlagen. Zur Ursachensuche erfolgte eine NaviTCD.

Abbildung 12 zeigt die Dopplerkurven der MCA (A) und PCA (B) mit der zugehörigen Navigationsbildgebung. Das Target liegt im M1-Segment der MCA, an dem eine mittlere Flussgeschwindigkeit von $156 \mathrm{~cm} / \mathrm{s}$ abgeleitet wurde. In einer Entfernung von lediglich 5,0 mm (entspricht einer Achsabweichung des Schallstrahls um etwa vier Grad) wurde ein Signal der PCA erfasst (mean 68 cm/s). Die Abbildung 13 zeigt einen Dopplerausdruck (mean 84 $\mathrm{cm} / \mathrm{s}$ ), der mittels konventioneller Dopplersonographie erfasst wurde. Das gezeigte Flusssignal wurde vom Untersucher als Signal der linken MCA in 55mm Messtiefe interpretiert.

Vergleicht man diese Kurvencharakteristik mit der Kurve der PCA (Abb. 12, B), so wird deutlich, dass sich beide Kurven sehr ähneln. Folglich wurde am wahrscheinlichsten das Dopplersignal der PCA erfasst und fälschlicherweise dem M1-Segment der MCA zugeordnet. 

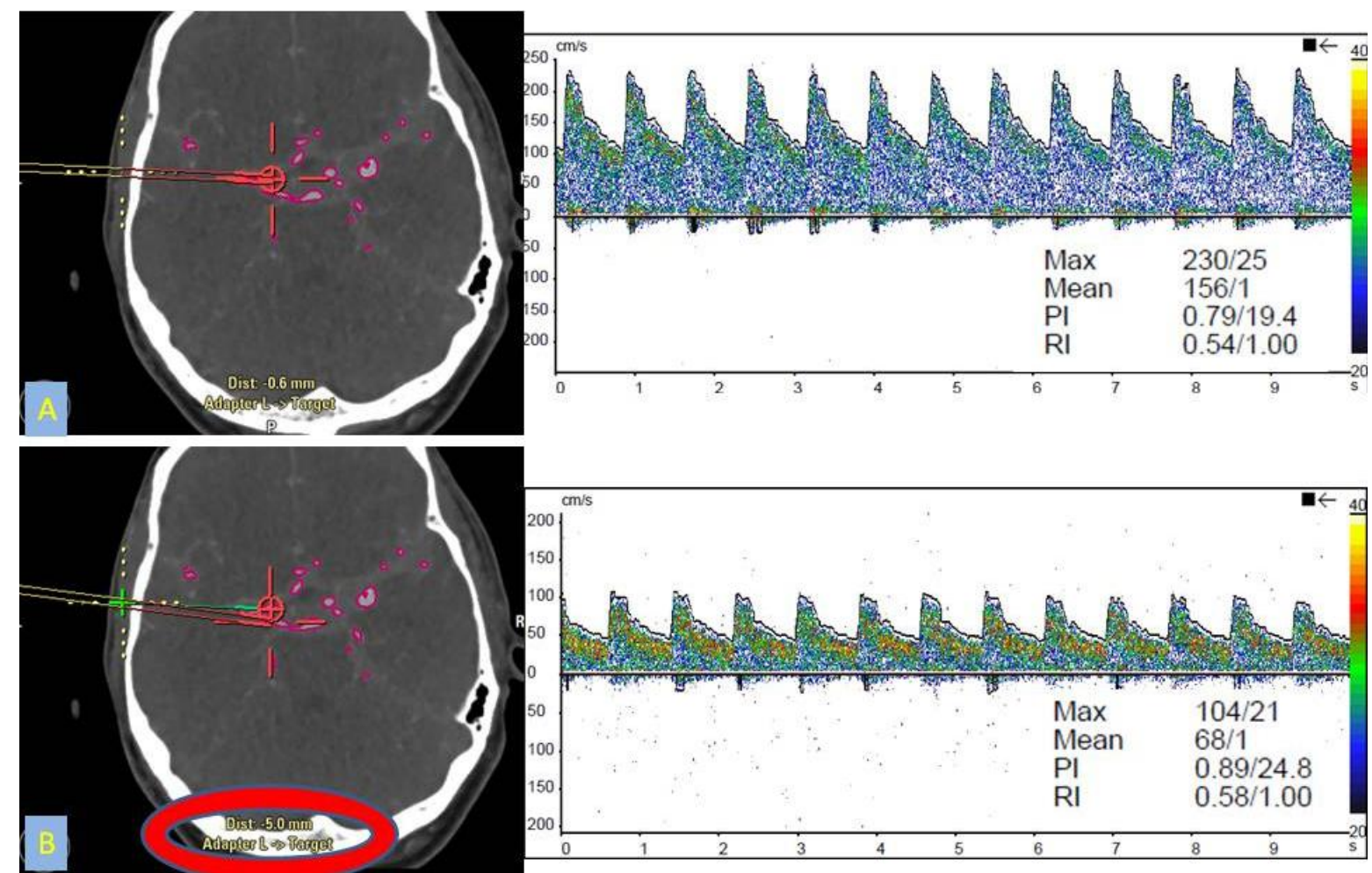

Abb. 12: A: axiales CT-A-Navigationsbild mit dem Target in der linken MCA und korrespondierendem Dopplersignal. B: axiales Navigationsbild und Dopplerkurve. Die Sonde insoniert die PCA, die $5 \mathrm{~mm}$ entfernt nahezu parallel zur MCA verläuft.

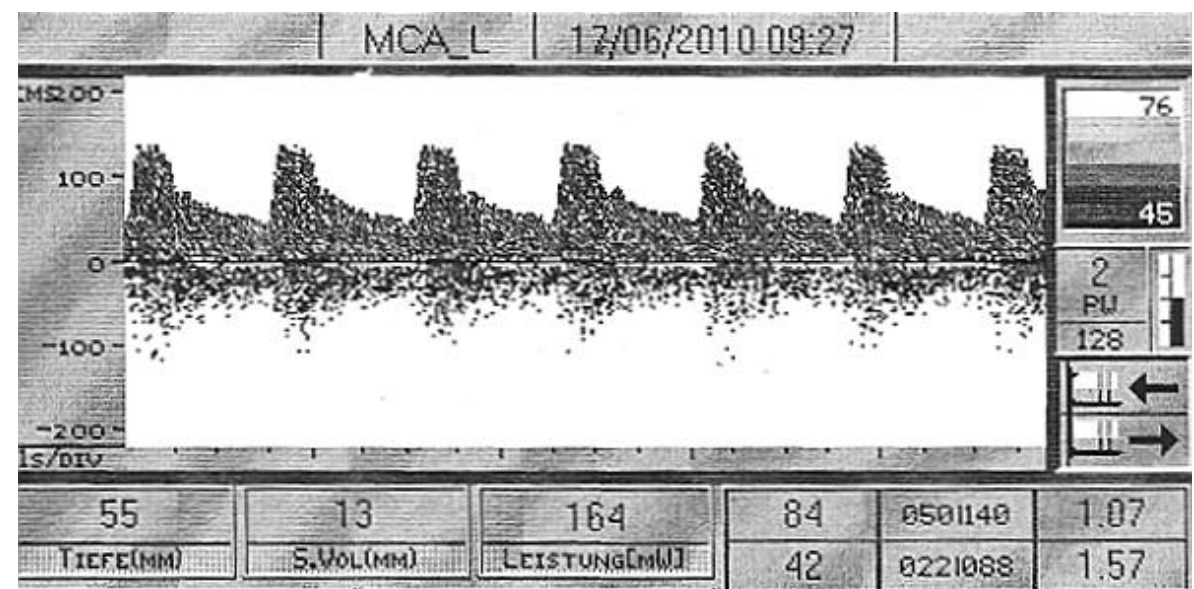

Abb. 13: konventionell gemessenes Dopplersignal. Das Signal wurde vom Untersucher als ,MCA_L“ benannt. 


\subsection{Räumliche Genauigkeit}

Da nach allein neuronavigiertem Ausrichten der Ultraschallsonde (d.h. ohne Berücksichtigung des Dopplersignals) nicht selten durch geringe Korrektur der Sondenachse ein verbessertes Signal erfassbar war, wurden im Folgenden die Distanzen zwischen den jeweiligen Punkten des vordefinierten Gefäßtargets und dem Ort des besten ableitbaren Flusssignals gemessen (Abb. 14), um ein Maß für die räumliche Genauigkeit der nichtinvasiven navigierten TCD zu erhalten. Nach Auswertung der 99 Messungen, von denen 94 auswertbar waren, ergab sich eine mittlere räumliche Distanz von $2,64 \mathbf{~ m m}( \pm \mathbf{1 , 1 5}$ $\mathbf{m m})$.

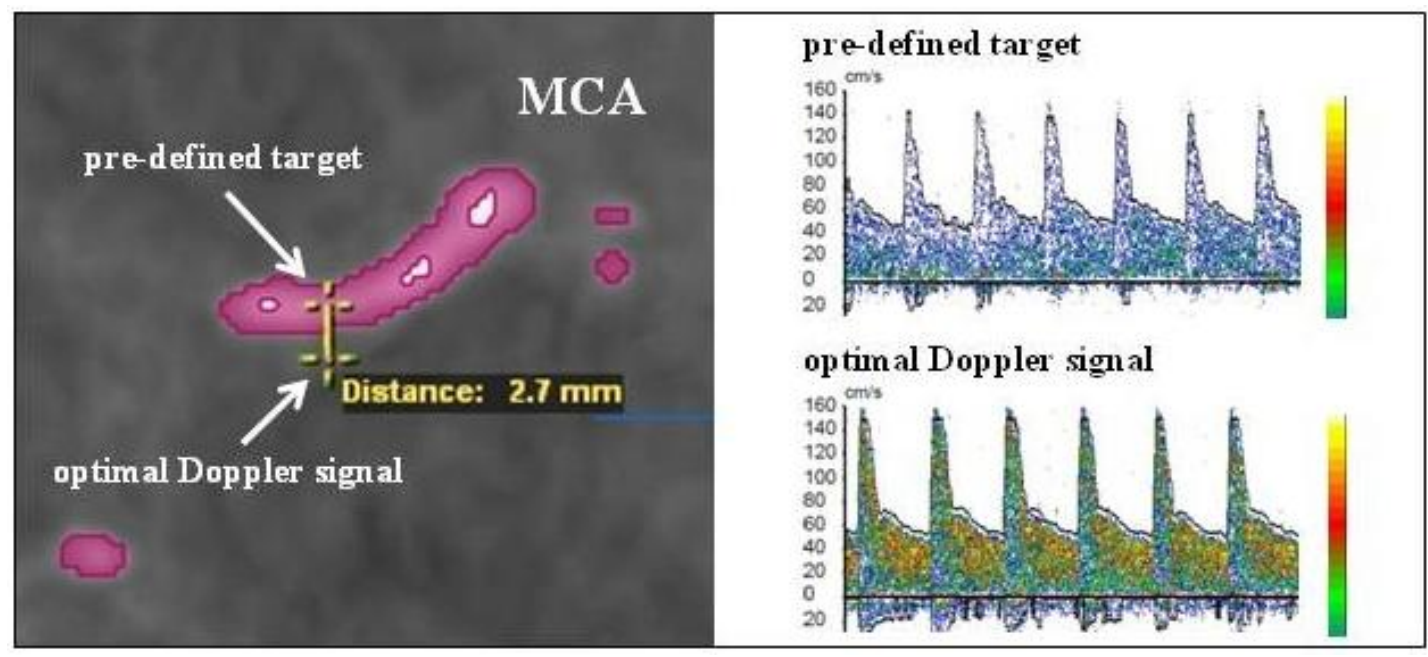

Abb. 14: Messung der Distanz zwischen geplantem Messpunkt und der Koordinate, an der das beste Dopplersignal abgeleitet werden konnte. 
Tabelle 4 zeigt detailliert die Ergebnisse für die Gefäßsegmente MCA, ICA-T und BA-Kopf.

\begin{tabular}{|c|c|c|c|c|}
\hline Insoniertes Gefäßsegment & MCA & ICA-T & BA-Kopf \\
$(\mathrm{n}=33)$ & $(\mathrm{n}=32)$ & Gesamt \\
$(\mathrm{n}=29)$ & 2,99 & 2,36 & $\mathbf{2 , 6 4}$ \\
\hline Mittelwerte Distanzen $(\mathrm{mm})$ & 2,54 & 1,14 & 1,01 & $\mathbf{1 , 1 5}$ \\
\hline Standardabweichung $(\mathrm{mm})$ & 1,23 & 2,99 & \\
\hline
\end{tabular}

Tab. 4: Mittelwerte der Distanzen der Koordinaten vom Target zum Punkt des besten Flusssignals.

Um zu überprüfen, ob Messungen aus seriellen Untersuchungen, vergleichbare Ergebnisse liefern, wurden in einer weiteren experimentellen Serie die Koordinaten aus Wiederholungsuntersuchungen, wie sie auch im Verlauf nach SAB erforderlich werden (vgl. S. 4, Kap. 2.2.1.1), ausgewertet. Dabei wurde in der initialen Untersuchung die Koordinate des besten Messsignals erfasst und die Distanz zum Punkt in der Wiederholungsuntersuchung gemessen. Insgesamt wurden 19 Wiederholungsuntersuchungen an acht Patienten durchgeführt. Dabei ergab sich an 56 Messpunkten (an einem Punkt kein messbares Signal (1,96\%)) eine mittlere Distanz von $\mathbf{2 , 7 5}$ mm mit einer Standardabweichung von 1,20 mm. 
Im Einzelnen waren die Werte folgendermaßen verteilt:

\begin{tabular}{|c|c|c|c|c|}
\hline Insoniertes Gefäßsegment & MCA & ICA-T & BA-Kopf \\
$(\mathrm{n}=19)$ & $(\mathrm{n}=19)$ & $\begin{array}{c}\text { Gesamt } \\
(\mathrm{n}=18)\end{array}$ & 2,90 & $\mathbf{2 , 7 5}$ \\
\hline Mittelwerte Distanzen $(\mathrm{mm})$ & 2,64 & 2,71 & 1,44 & $\mathbf{1 , 2 0}$ \\
\hline Standardabweichung $(\mathrm{mm})$ & 1,05 & 1,17 & 2,90 \\
\hline
\end{tabular}

Tab. 5: Mittelwerte der Distanzen der Koordinaten vom Punkt des besten Signals der ersten Untersuchung zum Punkt des besten Signals in einer Wiederholungsuntersuchung.

\section{Fallillustration Vasospasmusmonitoring}

Häufige Anwendung findet die TCD zum Monitoring von Blutflussgeschwindigkeiten nach subarachnoidalen Blutungsereignissen, um Vasospasmen frühzeitig zu erkennen (Rigamonti et al. 2008).

Im folgenden Fall wurde die neuronavigierte TCD eingesetzt, um die Veränderung von Flussgeschwindigkeiten und somit mögliche Kaliberveränderungen der basalen Hirnarterien bei einem 65-jährigen Patienten zu detektieren, der eine Ruptur eines Aneurysmas an der Bifurkation der rechten MCA erlitten hatte. Nachdem das Vorliegen von Vasospasmen angiographisch („Goldstandard“ (Willinsky et al. 2003)) gesichert wurde, wurde im Untersuchungsverlauf mehrmals therapeutisch interveniert. Der Erfolg dieser Intervention konnte auch anhand der Messwerte der navigierten TCD beobachtet werden. Die NaviTCD wurde nicht $\mathrm{zu}$ diagnostischen Zwecken, sondern lediglich ergänzend zur täglich durchgeführten konventionellen Untersuchung angewandt. 
$\mathrm{Zu}$ Beginn der Untersuchungsreihe wurde navigiert das Flusssignal des distalen M1Segmentes der rechten MCA erhoben und am Ort des besten Signals (Messtiefe $59 \mathrm{~mm}$ ) eine Trajektorie akquiriert. $\mathrm{Zu}$ diesem Zeitpunkt war eine gefährlich erhöhte mittlere Flussgeschwindigkeit von $254 \mathrm{~cm} / \mathrm{s}$ messbar (vgl. Abb. 15, B). Trotz dieses Befundes konnte aufgrund des schlechten kardio-pulmonalen Zustandes des Patienten zu diesem Zeitpunkt keine medikamentöse oder angioplastische Therapie begonnen werden.
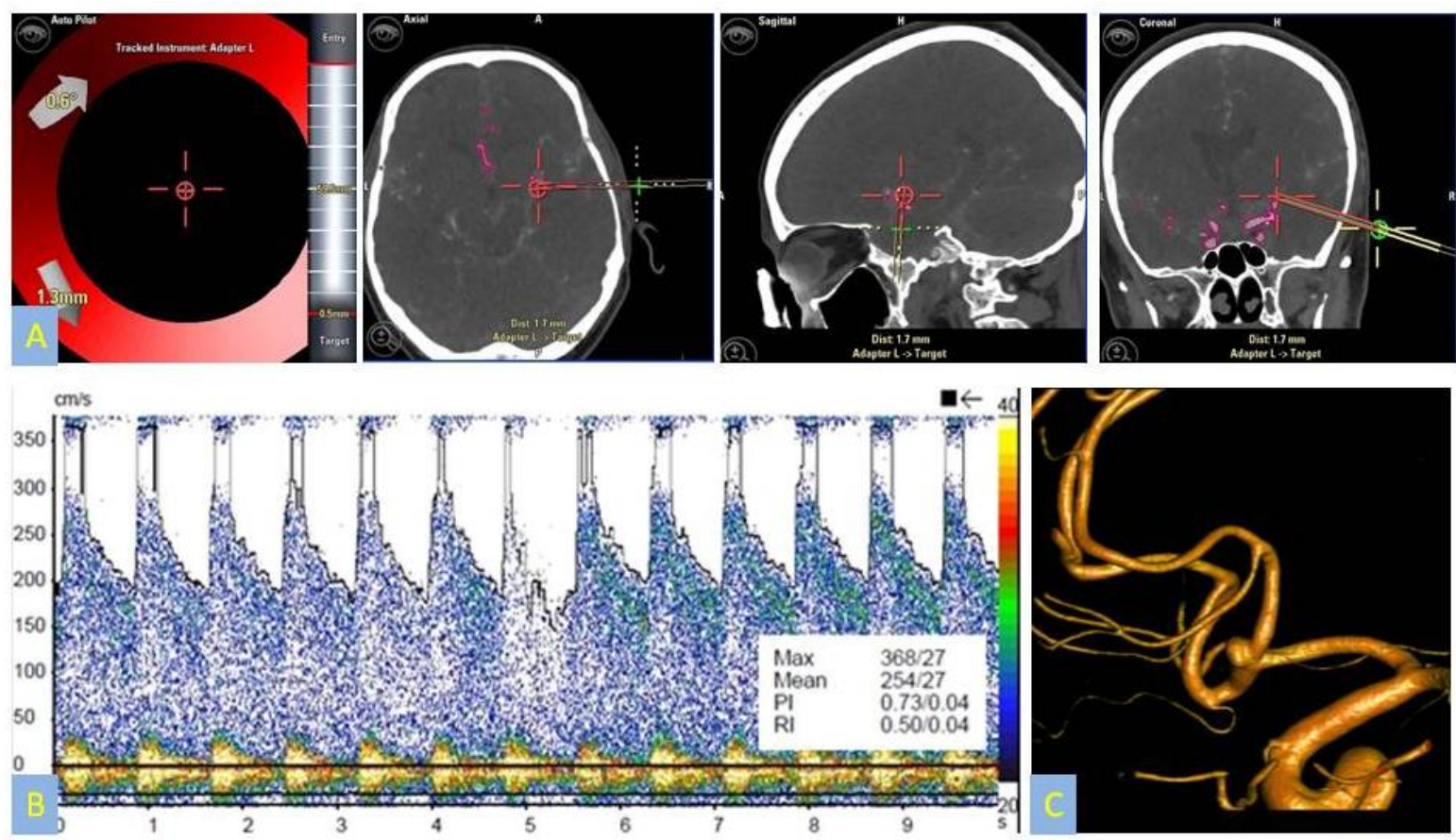

Abb. 15: A: Trajektorie mit Target am Ort des optimalen Signals für die distale MCA. Die Autopilotfunktion (links) hilft dem Untersucher beim korrekten Positionieren und Ausrichten der Ultraschallsonde. B: korrespondierendes Dopplersignal. C: digitale

Subtraktionsangiographie mit einem Aneurysma an der Bifurkation der rechten MCA.

Unter Zuhilfenahme der Trajektorie und der Autopilot-Funktion konnten die Flussgeschwindigkeiten stets zielgenau im gleichen Gefäßsegment (im Rahmen der in diesem Kapitel genannten Genauigkeit) im Verlauf von neun Tagen und fünf Wiederholungsuntersuchungen gemessen werden. Am ersten Tag (Tag drei nach dem 
Blutungsereignis) wurde, wie oben bereits erwähnt, eine mittlere Flussgeschwindigkeit von $254 \mathrm{~cm} / \mathrm{s}$ gemessen. Auch am Folgetag wurden ähnliche Werte beobachtet. In einer späteren dritten Untersuchung (nach lokal-medikamentös-interventioneller Angiographie mit Nimodipin) zeigten sich erniedrigte mittlere Flussgeschwindigkeiten von $186 \mathrm{~cm} / \mathrm{s}$. Die am siebten Tag nach dem Blutungsereignis durchgeführte vierte Messung ergab wiederum erhöhte Werte von $274 \mathrm{~cm} / \mathrm{s}$. Die anschließend begonnene systemische medikamentöse Therapie mit Nimodipin spiegelte ihren Erfolg auch in den TCD-Messwerten wider. So sanken die Flussgeschwindigkeiten bis zur fünften Messung wieder auf $186 \mathrm{~cm} / \mathrm{s}$.

Diese Fallillustration zeigt, wie Trajektorien zusammen mit der Autopilotfunktion eingesetzt werden können, um spezifische Gefäße zuverlässig stets im selben Segment und im seriellen Verlauf zu untersuchen.

\subsection{Genauigkeit im Verlauf der Untersuchung}

$\mathrm{Ob}$ die Referenzierung von intensivmedizinischen Patienten mithilfe der unter 3.3.1 beschriebenen nicht-invasiven Registrierung (mit Verwendung eines Kopfbandes) eine hinreichende Genauigkeit über die gesamte Untersuchungsdauer und unter Bedingungen der intensivmedizinischen Versorgung von Patienten erzielt, wurde durch Akquirierung von drei intra-prozedural angelegten Koordinaten untersucht. Deren Koordinaten wurden jeweils zu Beginn und am Ende der Untersuchung erfasst und verglichen. Hätte sich das Kopfband während der Untersuchung disloziert, so wäre die Raumkoordinate des Klebemarkers nach der Untersuchung verschieden von der vor der Untersuchung gewesen. Als Kontrolle wurden 18 Registrierungen unter nicht-intensivmedizinischen Kautelen an einem freiwilligen gesunden Probanden durchgeführt, anschließend drei Punkte akquiriert und deren Koordinate 
ohne Zeitverzug erneut bestimmt. Hierfür ergab sich eine mittlere Abweichung von $1.46 \pm$ 0.24 mm ( $\mathrm{n}=18$; Dauer: <1 min.). Die Stabilität der Registrierung unter intensivmedizinischen Bedingungen wurde in 31 Patientenuntersuchungen gemessen. Im Durchschnitt dauerten diese Untersuchungen 25,6 \pm 17,8 min (vgl. Abb. 16). Die längste Untersuchung dauerte 61 Minuten, die kürzeste hingegen vier Minuten.

Nach Auswertung der 93 Koordinaten ergab sich eine mittlere räumliche Abweichung von $\mathbf{1 , 5 1} \mathbf{m m} \pm \mathbf{0 , 4 4} \mathbf{m m}(\max .3,2 \mathrm{~mm} / \min .0,3 \mathrm{~mm})$. Der Unterschied zwischen den verschiedenen Untersuchungsbedingungen war somit nicht statistisch signifikant $(p=0,67)$.

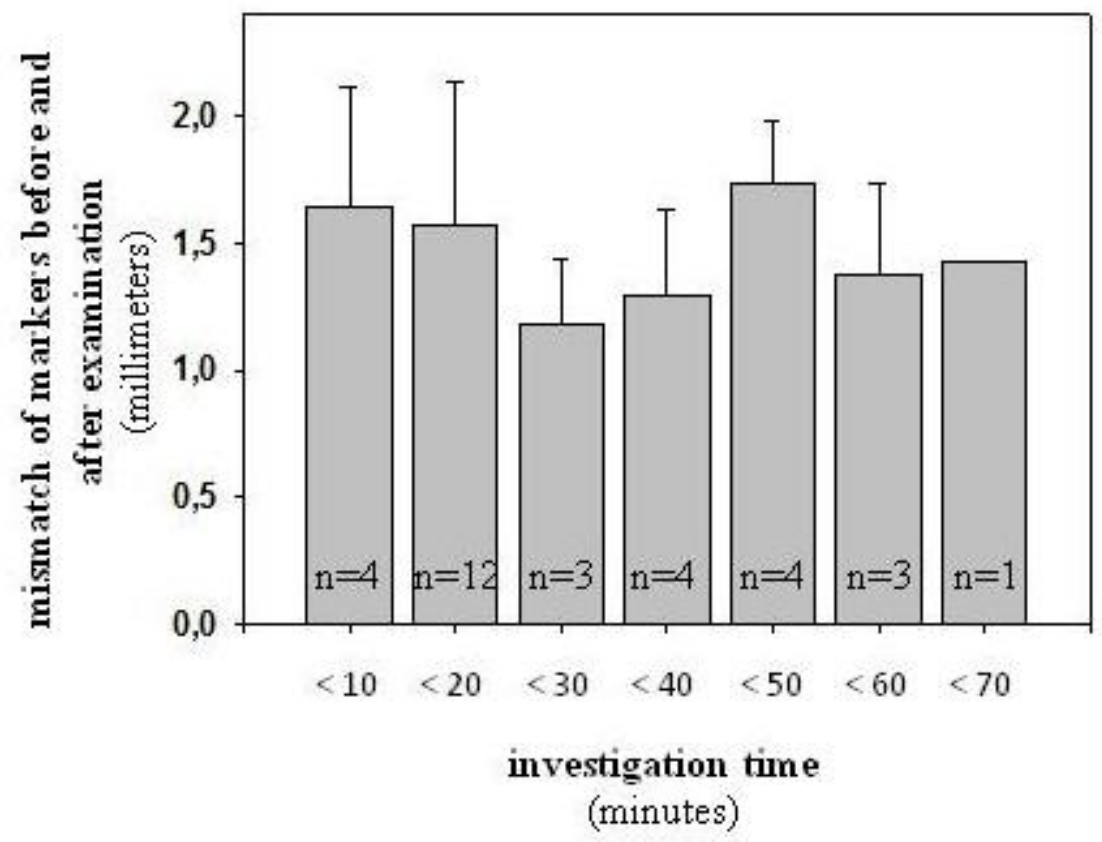

Abb. 16: Koordinatenänderung bei Wiederaufsuchen der Klebemarker am

Untersuchungsende in Abhängigkeit von der Untersuchungsdauer. 


\subsection{Abhängigkeit der Ergebnisse vom Alter des CT-A-Datensatzes}

Alle CT-A wurden zeitlich unabhängig von der TCD-Untersuchung erstellt und stellen nur eine Momentaufnahme der zerebralen Anatomie dar. Um eine möglicherweise daraus entstandene Ungenauigkeit zu überprüfen, wurde die räumliche Genauigkeit dem Alter des Schichtbilddatensatzes gegenübergestellt.

Detailliert aufgetragen nach der Zeit, die zwischen Erstellung des CT-A-Datensatzes und NaviTCD-Untersuchung verging, zeigt die folgende Tabelle die mittlere Distanz von der Koordinate des prä-definierten Punktes, an dem das beste Signal erwartet wurde (vgl. S. 26, Kap. 3.3.1) zu dem Punkt, an dem das beste Signal tatsächlich abgeleitet wurde.

\begin{tabular}{|c|c|c|c|}
\hline $\begin{array}{c}\text { Zeit von CT-A bis } \\
\text { NaviTCD }\end{array}$ & $\begin{array}{c}\text { Mittlere Distanz } \\
(\mathbf{m m})\end{array}$ & $\begin{array}{c}\text { Standard- } \\
\text { abweichung }\end{array}$ & $\mathbf{n}$ \\
\hline 0 - 2 Tage & 2.46 & 0.756 & 6 \\
\hline 3 - 5 Tage & 2.11 & 1.022 & 6 \\
\hline 6 - 10 Tage & 2.61 & 0.743 & 2 \\
\hline 10 - 14 Tage & 3.42 & 0.311 & 4 \\
\hline 15 - 30 Tage & 3.19 & 1.218 & 5 \\
\hline$>$ 30 Tage & 2.75 & 0.656 & 7 \\
\hline
\end{tabular}

Tab. 6: Distanzen in Abhängigkeit vom Alter der CT-Angiographie

Vergleicht man die Gruppe der Untersuchungen mit einem CT-A-Alter größer als 30 Tage $(n=5)$ mit denen, in denen der Datensatz maximal zwei Tage alt war $(n=6)$, so ergibt sich kein statistisch signifikanter Zusammenhang $(\mathbf{p}=\mathbf{0 , 5 1})$ zwischen beiden Gruppen. 


\section{Diskussion}

Die transkranielle Dopplersonographie ist ein nichtinvasives Verfahren zum Monitoring von Blutflussgeschwindigkeiten in den basalen Hirnarterien und somit indirekt auch zur Detektion von Gefäßspasmen. Die Höhe der Veränderung steht dabei in einer inversen Beziehung zum Gefäßkaliber (Aaslid 2002). Die Vorteile der Methode liegen darin, dass es sich um ein nichtinvasives Verfahren handelt, das kostengünstig und beliebig häufig am Patientenbett wiederholbar ist (Rigamonti et al. 2008). Dies hat dazu geführt, dass es sich als Monitoringverfahren auf neurochirurgischen Intensivstationen etablieren konnte (Tsivgoulis et al. 2009). Allerdings handelt es sich bei der TCD um eine relativ zeitaufwendige Technik, deren Verwertbarkeit und Reproduzierbarkeit durch den Ausbildungsstand und die Routine des Untersuchers bestimmt wird (Kremser et al. 1999).

Zur Zuordnung eines Dopplersignals zum anatomischen Korrelat kann bisher nicht auf eine Bildgebung oder anatomische Referenz zurückgegriffen werden. Lediglich einige Zuordnungskriterien können dem Untersucher helfen, Rückschluss auf das insonierte Gefäß zu ziehen. Häufige Varianten der normalen Anatomie und Kontraindikationen zu arteriellen Kompressionstests können die sichere Zuordnung erschweren (Grolimund et al. 1987) (vgl. S.10, Kap. 2.2.2.2, Gefäßidentifikation).

Die Idee, die TCD um eine Navigationstechnik zu erweitern, wurde 1999 erstmals von Auer, Kremser und Kollegen aufgegriffen (Auer et al. 1999, Kremser et al. 1999). Als Navigationsgrundlage diente ihnen ein zum Zwecke der TCD-Untersuchung angefertigter 3DMRT-Datensatz. Zur Probandenregistrierung wurde eine mit Referenzmarkern bestückte Gesichtsmaske verwendet, die dem Probanden vor der MRT-Untersuchung angelegt werden musste. Die anatomisch reproduzierbare Fixation der Maske am Kopf gelang mittels eines 
vorher angefertigten Oberkiefer-Gebissabdrucks und einer Fixierung an der Nasenwurzel mit Kopfbändern (Kremser et al. 1999).

Der Fortschritt bei Hard- und Software sowie der flächendeckende Einsatz von Neuronavigationsgeräten in der Neurochirurgie lässt es heute zu, auch ohne aufwendige Techniken den Patientenkopf nichtinvasiv und reproduzierbar mit bereits vorhandenen Bilddatensätzen zu referenzieren. Hierin besteht der wesentliche Unterschied der in dieser Studie verwendeten Methode zur Methode von Auer et al. Während in Auers Untersuchungsablauf dem Patienten die Referenzmaske noch vor Beginn der MRTUntersuchung angelegt werden musste, so konnte in dieser Studie der Kopf mithilfe einer nichtinvasiven Landmarkenregistrierung referenziert werden. Dadurch wurde es möglich, CTAufnahmen zu verwenden, die völlig unabhängig von der TCD-Untersuchung erstellt worden waren. Dabei ist prinzipiell jede Art von Schichtbildaufnahme geeignet, sofern sie zum hier verwendeten Navigationsgerät kompatibel ist (vgl. S,18, Kap. 2.3.1). Da die meisten neurochirurgischen Patienten, besonders solche, bei denen ein TCD-Monitoring erforderlich wird, im Krankheitsverlauf zu diagnostischen Zwecken CT- oder MRT-Bildgebungen erhalten, müssen sie sich keiner zusätzlichen radiologischen Untersuchung unterziehen (Kantelhardt et al. 2011).

Die Kombination aus Landmarkenregistrierung, Nutzung eines Kopfbandes und Verwendung von bestehenden CT-Aufnahmen erklärt die Nichtinvasivität der Methode. So muss der Patient zu keinem Zeitpunkt einer weiteren, potentiell schädlichen, ionisierenden Strahlenbelastung ausgesetzt werden oder sonstiger invasiver Maßnahmen zur Referenzierung (z.B. Anbringen einer Mayfield-Klemme) unterzogen werden. Auch der logistische, zeitliche und wirtschaftliche Aufwand zur Anfertigung neuer Schichtbildaufnahmen entfällt. 
Die Ergebnisse dieser Studie zeigen, dass die hier demonstrierte Navigationsmethode eine sinnvolle und genaue Ergänzung zur konventionellen transkraniellen Dopplersonographie sein kann. Im Durchschnitt war das beste Flusssignal 2,64 $\mathrm{mm}( \pm 1,15 \mathrm{~mm})$ vom Zentrum des bildmorphologischen Gefäßkalibers der Navigationsbildgebung entfernt ableitbar. Da in der Mehrheit der regelmäßig durchgeführten konventionellen TCD ein Sample Volume mit einem Durchmesser von sechs bis acht Millimetern gewählt wird (Nicoletto und Burkman 2009), kann davon ausgegangen werden, dass regelhaft am Navigationstarget ein korrespondierendes Signal ableitbar sein wird, was durch die Ergebnisse dieser Untersuchung bestätigt wird. Vergleicht man die Koordinaten des besten Messsignals aus der primären Untersuchung mit denen aus Folgeuntersuchungen, so zeigte sich, dass die Punkte im Mittel um 2,76 mm $( \pm 1,2$ mm) deviierten. In der Literatur wurden für die Genauigkeit der rahmenlosen Neuronavigation ähnliche Werte publiziert, wie sie in dieser Studie ermittelt wurden. Eine Veröffentlichung über die Genauigkeit der Neuronavigation für die Platzierung von Stimulationselektroden (präoperative Planung als Trajektorie) ergab eine mittlere Abweichung von $3 \mathrm{~mm}( \pm 1,5 \mathrm{~mm})$ (Mascott 2006). Ähnliche Ergebnisse belegte auch eine weitere publizierte Studie (Giese et al. 2010). Mit 4,05 $\mathrm{mm}( \pm 3,62 \mathrm{~mm})$ zeigten Muacevic et al. einen etwas größeren Fehler (Muacevic et al. 2000). Bei allen genannten Untersuchungen muss allerdings berücksichtigt werden, dass sie sich auf offen-chirurgische Eingriffe beziehen, bei denen (anders als in NaviTCD Studie) ein Brain-Shift durch Eröffnung der Kalotte, Substanzdefekte und Liquorverlust als zusätzliche Fehlerquelle in Frage kommt. Da Ultraschallwellen beim Durchtritt durch Knochen gestreut werden (Evans 2006), muss eine Achsabweichung des Schallstrahls bei der Verwendung von TCD zusammen mit Neuronavigation als weitere Fehlerursache in Betracht gezogen werden. Aus der ermittelten 
Genauigkeit $(2,64 \pm 1,15 \mathrm{~mm})$ lässt sich allerdings schließen, dass dieser Fehler nur einen geringen Einfluss zu haben scheint.

Dennoch kann durch die erhobenen Daten angenommen werden, dass die in dieser Studie angewandte nichtinvasive Methode zur Referenzierung des Navigationsdatensatzes mit anatomischen Landmarken eine vergleichbare Genauigkeit und Zuverlässigkeit erzielt wie die konventionelle (Fiducial-based paired-point registration) Methode (siehe S. 17, Kap. 2.3.1).

Kritisch bleibt in diesem Zusammenhang allerdings zu diskutieren, dass eine CT-A nur eine Momentaufnahme der zerebralen Anatomie liefert. Mögliche Raumforderungen (z.B. durch fortschreitende Blutungen) oder Parenchymverschiebungen nach Kraniotomie (sogenannter Brain-Shift) könnten die Genauigkeit und Reproduzierbarkeit der Navigation beeinflussen. Obwohl dieser Einfluss in dieser Studie nicht beobachtet werden konnte, kann die wahre Bedeutung dieser Fehlerquelle nur durch weitere Studien geklärt werden.

Angesichts des Umstandes, dass der Referenzstern in dieser Untersuchung nicht invasiv am Kopf angebracht wurde, sondern mit einem flexiblen Kopfband fixiert wurde, war potentiell nicht auszuschließen, dass das Kopfband während der Untersuchung verrutschen könnte und somit die Genauigkeit der Navigation nicht mehr gewährleistet wäre. Um diese Fehlermöglichkeit zu untersuchen, wurde, wie unter 4.3 erläutert, die Genauigkeit der Registrierung über die Zeit der Untersuchung evaluiert. In der Auswertung von 31 Untersuchungen (durchschnittliche Dauer 25,6 min \pm 17,8 $\min$ (min. vier min / $\max .61 \mathrm{~min}$ )) zeigte sich, dass in keiner der unter intensivmedizinischen Bedingungen durchgeführten Untersuchungen eine wesentliche Verschiebung des Kopfbandes stattgefunden hatte und dadurch kein Einfluss auf die Genauigkeit der Registrierung bestand. Ebenso war kein 
signifikanter Zusammenhang zwischen Dauer der Untersuchung und einer Ungenauigkeit der Registrierung zu erkennen $(\mathrm{p}=0,67)$

Aufgrund der oben beschriebenen regelmäßigen Zuverlässigkeit der nichtinvasiven Referenzierung ist es möglich, in einer primären Untersuchung Trajektorien (=Vektoren) zu akquirieren, die den Eintrittsort des Ultraschalls durch den Schädelknochen und ein Gefäßtarget im Gehirn definieren. Da hierdurch Position, Messtiefe und Richtung der Schallachse vorgegeben sind, können die Trajektorien in Verbindung mit dem Navigationsautopiloten in Wiederholungsuntersuchungen dazu genutzt werden, um reproduzierbar dasselbe Gefäßsegment im gleichen Winkel zu insonieren. Somit kann sowohl die Abhängigkeit von Routine und Ausbildungsstand des Untersuchers als auch die Inter- und Intra-Untersuchervariabilität reduziert werden (Kantelhardt et al. 2011).

Die zur navigierten Doppleruntersuchung verwendeten CT-Datensätze wurden nicht zum Zweck der TCD angefertigt und standen somit auch in keinem direkten zeitlichen Zusammenhang. Um zu überprüfen, ob das Alter des Datensatzes einen Einfluss auf die Genauigkeit haben könnte, wurden, wie unter 4.4 gezeigt, die Ergebnisse in Abhängigkeit vom Alter der CT-A aufgetragen. Bei der Gegenüberstellung der Gruppe „CT Alter $<3 \mathrm{~d}^{\text {“ mit }}$ der Gruppe „CT Alter > 30d“ zeigte sich jedoch kein statistischer Zusammenhang zwischen Alter der CT-A und Ungenauigkeit der NaviTCD ( $p=0,51)$. Somit spielt es offenbar für die Durchführbarkeit einer NaviTCD eine geringe Rolle, wann der Datensatz erstellt wurde. In einer einzelnen Untersuchung, die nicht in die Auswertung dieser Arbeit einbezogen wurde, konnte gezeigt werden, dass auch die Verwendung einer 161 Tage alten CT-Angiographie zuverlässige und reproduzierbare TCD-Ergebnisse liefert. 
Allerdings muss berücksichtigt werden, dass bei dieser Gegenüberstellung eventuell stattgehabte Raumforderungen nicht berücksichtigt wurden. Möglicherweise haben massive spätere intrakranielle Raumforderungen einen Einfluss auf die Lage der basalen Hirnarterien, was es notwendig machen kann, einen aktualisierten Datensatz zu verwenden. Bei vielen Patienten in neuro-intensivmedizinischer Therapie werden allerdings regelhaft aktuelle CT, CT-A oder MRT-Untersuchungen zu diagnostischen Zwecken notwendig.

Das Fallbeispiel im Kapitel 5.1 illustriert, dass für die postoperative Patientenüberwachung auf Neurointensivstationen mithilfe der intra-prozedural planbaren Navigationstrajektorien Untersuchungsprotokolle erstellt werden können. Während der ersten Untersuchung wurde eine Trajektorie angelegt, die zum einen den Ort, an der der Schädelknochen vom Ultraschall penetriert werden konnte und zum anderen einen fixen Messpunkt im distalen M1-Segment der A. cerebri media definierte. Durch Verwendung des Kolibri-Autopiloten ergaben sich in den vier Folgeuntersuchungen stets vom Untersucher unabhängige, objektive Messergebnisse, weil sowohl Einschalltiefe als auch Einschallwinkel als Variablen entfielen.

Kritisch in dieser Hinsicht ist zu betrachten, dass die Neuronavigation keine uneingeschränkte Genauigkeit erreicht. Es konnte gezeigt werden, dass für die räumliche Auflösung ein Wert von durchschnittlich $2,64 \mathrm{~mm}( \pm 1.15 \mathrm{~mm})$ für die Entfernung zwischen dem geplanten Target und dem Ort des besten abgeleiteten Messsignals erreicht wurde (siehe S. 35, Kap. 4.2). Kleine Nachjustierungen der Sondenposition können also gelegentlich notwendig sein, um ein optimales Messsignal abzuleiten. Aufgrund der relativ geringen Ungenauigkeit im Zehntel-Zentimeterbereich sollte jedoch nicht davon auszugehen sein, dass diese Nachjustierung einen entscheidenden Einfluss auf den Einschallwinkel und somit auf die Höhe der gemessenen Flussgeschwindigkeit haben wird. 
Zusätzlich zur Feststellung, dass Navigationstrajektorien objektivere Ergebnisse liefern als Freihand-TCD-Untersuchungen, kann die NaviTCD den Zeitaufwand bei Folgeuntersuchungen deutlich verringern (Kantelhardt et al. 2011). Trotz der Entwicklung des Doppler-M-Modes, der dem Untersucher Flusssignale entlang der gesamten Ultraschallachse anzeigt und somit die Schallfenstersuche erleichtert (Alexandrov et al. 2002), geht nicht selten einige Zeit dadurch verloren, dass zu Beginn einer Untersuchung zunächst ein geeignetes Schallfenster durch „Probieren“ gefunden werden muss (Widder und Görtler, 2004). Einerseits kann die Navigation beim erstmaligen Suchen eines Schallfenster helfen, weil mithilfe des Navigationsgerätes der Schallstrahl zu jeder Zeit auf das Gefäß gerichtet werden kann und andererseits kann in Folgeuntersuchungen die Suche gänzlich entfallen, weil die Sondenposition an der Temporalschuppe (= Schallfenster) durch eine zuvor definierte Trajektorie bereits vorgegeben wird.

Ein grundsätzliches Problem bei der Anwendung transkraniellen Ultraschalls besteht darin, dass in einigen Fällen die transtemporale Beschallung erschwert oder gar unmöglich ist. Wie im Kapitel 2.2.2.1 beschrieben, sind fehlende Schallfenster mit einer Häufigkeit zwischen 5 und 10 Prozent realistisch. In Abhängigkeit von Alter, Geschlecht, Knochendicke und ethnischer Herkunft kann dieser Anteil jedoch auch höher sein (Wijnhoud et al. 2008, Yagita et al. 1996). Die NaviTCD kann dem Untersucher einerseits, wie oben beschrieben, helfen, Schallfenster zu finden und andererseits könnte auch die Beschallung über alternative Zugangswege ermöglicht werden. In einer Publikation von Lewis et al. wurde gezeigt, wie iatrogen erzeugte Knochendefekte (z.B. nach Aneurysma-Clipping) genutzt wurden, um die basalen Hirnarterien zu insonieren. Zur Detektion dieser Substanzdefekte wurde ein Neuronavigationsgerät mit herkömmlichen Pointer verwendet (Lewis et al. 2010). Es muss jedoch berücksichtigt werden, dass bei Nutzung solcher Zugangswege, aber auch anderer 
seltener "natürlicher" Schallfenster, abhängig von deren Lokalisation, eine anatomische Zuordnung des Dopplersignals deutlich erschwert sein kann. Ebenso hat der Einschallwinkel einen erheblichen Einfluss auf das Messergebnis (siehe S. 16, Kap. 2.2.2.3, Abb. 4B). Da bei Nutzung der NaviTCD sowohl eine anatomische Orientierung gegeben ist als auch eine Aussage über den Winkel gemacht werden kann, würde die Zuordnung von Flusssignal zum anatomischen Korrelat erleichtert und es könnte durch mathematische Korrektur die wahre Blutflussgeschwindigkeit ermittelt werden. Die NaviTCD könnte deshalb eine gute Ergänzung zur Idee von Lewis et al. darstellen.

In dieser Studie konnte in der initialen Untersuchung in allen Fällen ein adäquates Schallfenster gefunden werden. Ob dies der navigierten Hilfestellung geschuldet ist oder zufallsbedingt zu erklären ist, kann aufgrund des relativ kleinen Patientenkollektivs nicht eindeutig geklärt werden. Die Vermutung, dass durch Verwendung der Neuronavigation die Suche nach geeigneten Schallfenstern erleichtert und damit die Ausfallrate reduziert werden könnte, liegt allerdings nahe.

Die Beurteilung von Gefäßen, die einen pathologischen oder von der Normalanatomie abweichenden Verlauf aufweisen, war mit der TCD bisher nur eingeschränkt möglich und von der Erfahrung des Untersuchers abhängig. Die NaviTCD könnte zukünftig dabei helfen, auch im Fall von anatomischen Varianten oder Pathologien, die z.B. durch Gefäßmalformationen oder Tumorraumforderung entstehen, valide TCD-Ergebnisse zu erzielen.

Im Kapitel 2.2.3 (S. 16) wird erläutert, dass die Technik der transkranielle Duplexsonographie einige Vorteile gegenüber der Dopplersonographie aufweist. Da zur technischen Umsetzung Schallfrequenzen von $2-3 \mathrm{MHz}$ verwendet werden, unterliegt diese Methode allerdings 
ebenso dem Problem, dass schlechte oder nicht vorhandene Schallfenster die Untersuchung erschweren. Prinzip bedingt ist auch die geometrische Knochenfläche, die von den Schallwellen durchdrungen werden muss, beim TCCD größer (Evans 2006). Für die Anwendung der TCCD werden in der Literatur Ausfallraten von bis zu 10-20\% berichtet (Seidel et al. 1995, Droste 2008). Georgiadis und Kollegen zeigten, dass bei Verwendung von $1 \mathrm{MHz}-$ Ultraschallsonden die Ausfallquote für die TCD verringert werden kann (Georgiadis et al. 1999). Die Neuronavigation kann in Kombination mit 1- MHz-TCD eine sinnvolle nichtinvasive Alternative zur TCCD sein, da sowohl eine anatomische Bildgebung zur Verfügung steht als auch eine Winkelkorrektur möglich ist. Eine weitere Lösung, Ausfallraten durch insuffiziente Schallfenster $\mathrm{zu}$ reduzieren, besteht für beide Techniken in der Ultraschallkontrastmittelgabe (Totaro et al. 1999). Obwohl die Kontrastmittelgabe in der klinischen Anwendung weit verbreitet ist (Droste 2008), sollte aber bedacht werden, dass es sich hierbei um invasive Maßnahme handelt.

Wie oben bereits mehrmals erwähnt, hat der Insonationswinkel einen großen Einfluss auf die gemessenen Flussgeschwindigkeiten. Bisher war es ausschließlich mithilfe der TCCD möglich, eine exakte Winkelkorrektur durchzuführen (Martin et al. 1995). Manuelle Korrekturen mit der TCD beruhten bisher nur auf empirisch abgeschätzten Winkeln. Die navigierte TCD hingegen erlaubt es durch die Kenntnis des realen anatomischen Gefäßverlaufs, ebenso wie die TCCD, korrigierte und somit wahre Flussgeschwindigkeiten zu ermitteln. Da in der CT-A der Durchmesser des Gefäßes bestimmt werden kann, wäre es theoretisch überdies möglich, das tatsächliche Blutflussvolumen zu bestimmen (Kremser et al. 1999). Dabei bleibt jedoch zu berücksichtigen, dass die CT-A bzw. MR-A nur eine Momentaufnahme des Gefäßkalibers liefert und somit nur Näherungen bestimmt werden 
könnten. Ob die NaviTCD der TCCD in der Intensivmedizin gleichwertig oder gar überlegen ist, muss dennoch durch weiterführende Studien geklärt werden.

Trotz der überwiegend positiven Erfahrungen, die mit der NaviTCD gesammelt wurden, muss kontrovers über die Kosten dieses Verfahrens diskutiert werden. So sollte berücksichtigt werden, dass die Anschaffungskosten eines handelsüblichen Navigationsgeräts (z.B. Brainlab Kolibri®) je nach Ausführung das Fünf- bis Zehnfache eines Doppler-Geräts (z.B. DWL MultiDop T digital ${ }^{\mathrm{TM}}$ ) betragen.

Durch Erweiterung der TCD um ein Neuronavigationsverfahren kann die Anwender-bedingte Variabilität der TCD-Messungen verringert werden (siehe oben in diesem Kapitel) (Kantelhardt et al. 2011, Kremser et al. 1999). Diese Aussage setzt allerdings eine gute, reproduzierbare Genauigkeit der Navigation voraus. Die Navigationsgenauigkeit ist allerdings wiederum von der Referenzierung und somit von der anatomischen Kenntnis des Anwenders abhängig. Zur Registrierung ist es notwendig, die anatomischen Landmarken mittels des Pointers zu referenzieren. Ungenauigkeiten hierbei schlagen sich auf die Navigationsgenauigkeit und somit auch auf die TCD-Ergebnisse wieder. Solche Fehler lassen sich durch eine prä-prozedurale Plausibilitätsprüfung, wie sie in Kapitel 3.3.1 beschrieben wurde, allerdings weitestgehend minimieren. Dennoch sollte man stets im Hinterkopf behalten, dass auch die Neuronavigation einem Anwendungsfehler unterliegen kann (Grunert et al. 2003). 


\section{Ausblick}

Die Problematik der inadäquaten Schallfenster ist eines der Hauptprobleme sowohl der TCD als auch der TCCD. Grolimund et al. postulierten, dass die Schallabsorption von der temporalen Knochendicke abhängig sei (Grolimund et al. 1987). Somit könnte es möglich sein, dünne Knochenstellen und somit adäquate Schallfenster prädiktiv in der CT-Aufnahme $\mathrm{zu}$ erkennen, diese gezielt neuronavigiert aufzusuchen und dadurch den Zeitaufwand, der durch die Schallfenstersuche entsteht, weiter zu reduzieren.

Ferner ist in den vergangenen Jahren die Sonofibrinolyse ins Licht der SchlaganfallForschung gerückt. Aufgrund von Komplikationsereignissen hat sich die Methode allerdings noch nicht durchsetzen können (vgl. S.5, Kap. 2.2.1.2). Es ist denkbar, dass die NaviTCDMethode zur Reduktion der Komplikationen beitragen könnte, weil eine zielgenaue Insonation der Okklusionsstelle, ggf. auch mehrdimensional, quasi stereotaktisch, einfach realisierbar wäre. Der wirkliche Benefit der Sonothrombolyse muss dennoch zunächst durch weitere Studien geklärt werden.

Am Ende des Kapitels 5 wurden die hohen Anschaffungskosten für ein Neuronavigationsgerät diskutiert. Sollte sich die hier gezeigte Referenzierung künftig etablieren können (d.h. Verwendung eines flexiblen Kopfbandes anstatt einer MayfieldKlemme), so wären weitere Anwendungsbereiche für die Neuronavigation in der Akut- und Intensivmedizin denkbar (z.B. die Anlage von Ventrikeldrainagen) und die hohen Anschaffungskosten für den nicht-operativen Bereich würden sich aufgrund einer höheren Anwendungsvielfalt relativieren. 


\section{Zusammenfassung}

Die transkranielle Dopplersonographie (TCD) ist eine etablierte und weit verbreitete Technik zur Messung von Blutflussgeschwindigkeiten in den Hirnbasisarterien, die nicht invasiv ist und direkt am Patientenbett durchgeführt werden kann. Aufgrund der fehlenden Schichtbildgebung, wie sie z.B. die transkranielle Duplexsonographie (TCCD) bietet, ist die Gefäßidentifikation jedoch schwierig. Bei pathologischer oder embryonal-varianter Anatomie kann sie gar an ihre Grenzen stoßen. Ferner fehlt die Möglichkeit, eine exakte Winkelkorrektur der Messwerte durchzuführen, und in einigen Fällen ist die transkranielle Beschallung durch insuffiziente Schallfenster erschwert oder unmöglich.

Die hier dargestellten Ergebnisse und Fallbeispiele haben gezeigt, dass die Erweiterung der TCD um ein nichtinvasives Neuronavigationsverfahren (NaviTCD) aufgrund von guter Reproduzierbarkeit und Genauigkeit eine sinnvolle Ergänzung zur konventionellen Methode sein kann. Einerseits erleichtert die Navigation die anatomische Orientierung, andererseits kann der Beschallungswinkel bestimmt werden, was eine exakte Winkelkorrektur erlaubt, die bisher nur mit der TCCD möglich war. Die Insonation ausgehend von alternativen Schallfenstern war bisher nur eingeschränkt möglich, weil die Aussagekraft von typischen Identifikationskriterien, wie z.B. der Einschalltiefe, dadurch herabgesetzt ist. Mithilfe der NaviTCD kann eine sichere Zuordnung von Dopplersignal zum anatomischen Korrelat erfolgen.

Die weite Akzeptanz der TCD und TCCD zeigt, dass beide Techniken in vielen Fällen gute Ergebnisse liefern. Die NaviTCD kann auch dann als Alternative gesehen werden, wenn die konventionellen Methoden wegen Pathologien oder anderen erschwerenden Bedingungen versagen. Aufgrund der guten Reproduzierbarkeit der Messwerte kann die NaviTCD mit ihrer 
Autopilotfunktion aber auch dazu genutzt werden, Untersuchungsprotokolle für serielle Untersuchungen $\mathrm{zu}$ erstellen, wie sie z.B. für das Vasospasmusmonitoring nach subarachnoidalen Blutungsereignissen notwendig werden, um stets vom Untersucher unabhängige, valide und vergleichbare Messergebnisse zu erhalten. Weiterführende Studien sollten untersuchen, ob es möglich sein kann, anhand von CT-Aufnahmen prädiktiv adäquate Schallfenster zu bestimmen. Dadurch könnte die Zeit, die verloren geht, um ein Schallfenster $\mathrm{zu}$ finden, weiter reduziert werden. 


\section{Literaturverzeichnis}

Aaslid R (2002): Transcranial Doppler assessment of cerebral vasospasm. Eur J Ultrasound, $\underline{16}, 3-10$

Aaslid R, Markwalder TM, Nornes H (1982): Noninvasive transcranial Doppler ultrasound recording of flow velocity in basal cerebral arteries. J Neurosurg, 57, 769-774

Akamatsu Y, Kumabe T, Kanamori M, Nagamatsu K-ichi, Saito R, Sonoda Y, Tominaga T (2009): Intraoperative neuronavigation system without rigid pin fixation. No Shinkei Geka, $\underline{37}, 1193-1199$

Akdemir H, Oktem S, Menkü A, Tucer B, Tuğcu B, Günaldi O (2007): Image-guided microneurosurgical management of small arteriovenous malformation: role of neuronavigation and intraoperative Doppler sonography. Minim Invasive Neurosurg, 50, 163169

Alexandrov AV (2009): Ultrasound enhancement of fibrinolysis. Stroke, $\underline{40}, 107-110$

Alexandrov AV, Demchuk AM, Burgin WS (2002): Insonation method and diagnostic flow signatures for transcranial power motion (M-mode) Doppler. J Neuroimaging, 12, 236-244

Auer A, Felber S, Lutz W, Kremser C, Schmidauer C, Hochmair E, Aichner F (1999): Transcranial Doppler sonography guided by magnetic resonance angiography for improved monitoring of intracranial arteries. J Neuroimaging, $\underline{9}, 34-38$

Babikian VL, Feldmann E, Wechsler LR, Newell DW, Gomez CR, Bogdahn U, Caplan LR, Spencer MP, Tegeler C, Ringelstein EB et al (2000): Transcranial Doppler ultrasonography: year 2000 update. J Neuroimaging, $\underline{10}$, 101-115

Diehl RR, Berlit P: Funktionelle Dopplersonographie in der Neurologie. Springer, Berlin 1996

Doppler C: Ueber das farbige Licht der Doppelsterne und einiger anderer Gestirne des Himmels. Borrosch \& André, Prag 1842 
Dorsch NW (2011): A clinical review of cerebral vasospasm and delayed ischaemia following aneurysm rupture. Acta Neurochir, 110 Suppl, 5-6

Dorsch NW, King MT (1994): A review of cerebral vasospasm in aneurysmal subarachnoid haemorrhage Part I: Incidence and effects. J Clin Neurosci, 1 , 19-26

Droste DW (2008): Clinical utility of contrast-enhanced ultrasound in neurosonology. Eur Neurol, 59 Suppl 1, 2-8

Evans DH (2006): Physical and technical principles. Front Neurol Neurosci, 21, 1-18

Georgiadis D, Karatschai R, Uhlmann F, Lindner A (1999): Diagnostic yield of a 1-MHz transducer in evaluation of the basal cerebral arteries. J Neuroimaging, $\underline{9}, 15-18$

Gerriets T, Seidel G, Fiss I, Modrau B, Kaps M (1999): Contrast-enhanced transcranial colorcoded duplex sonography: efficiency and validity. Neurology, 52, 1133-1137

Giese H, Hoffmann K-T, Winkelmann A, Stockhammer F, Jallo GI, Thomale U-W (2010): Precision of navigated stereotactic probe implantation into the brainstem. J Neurosurg Pediatr, $\underline{5}, 350-359$

Grolimund P: Transmission of Ultrasound Through the Temporal Bone; in: Transcranial doppler sonography; hrsg. von Aaslid R; Springer, Wien 1986, 10-22

Grolimund P, Seiler RW, Mattle H (1987): Possibilities and limits of transcranial Doppler sonography. Ultraschall Med, $\underline{8}, 87-94$

Grunert P, Darabi K, Espinosa J, Filippi R (2003): Computer-aided navigation in neurosurgery. Neurosurg Rev, 26, 73-99

Gumprecht HK, Widenka DC, Lumenta CB (1999): BrainLab VectorVision Neuronavigation System: technology and clinical experiences in 131 cases. Neurosurgery, 44, 97-104

Itoh T, Matsumoto M, Handa N, Maeda H, Hougaku H, Hashimoto H, Etani H, Tsukamoto Y, Kamada T (1993): Rate of successful recording of blood flow signals in the middle cerebral artery using transcranial Doppler sonography. Stroke, 24, 1192-1195 
Jatuzis D, Zachrisson H, Blomstrand C, Ekholm S, Holm J, Volkmann R (2000). Evaluation of posterior cerebral artery blood flow with transcranial Doppler sonography: value and risk of common carotid artery compression. J Clin Ultrasound, 28, 452-460

Kantelhardt SR, Greke C, Keric N, Vollmer F, Thiemann I, Giese A (2011): Image Guidance for Transcranial Doppler Ultrasonography. Neurosurgery, $\underline{68}$, ons257-ons266

Kapoor K, Singh B, Dewan LIJ (2008): Variations in the configuration of the circle of Willis. Anat Sci Int, $\underline{83}$, 96-106

Keller H, Baumgartner G, Regli F (1973): Carotid artery stenoses and occlusions. Diagnosis by percutaneous ultrasound Doppler sonography at the supraorbital or supratrochlear artery. Dtsch Med Wochenschr, 98, 1691-1698

Khaffaf N, Karnik R, Winkler WB, Valentin A, Slany J (1994): Embolic stroke by compression maneuver during transcranial Doppler sonography. Stroke, 25, 1056-1057

Klötzsch C, Popescu O, Berlit P (1998): A new 1-MHz probe for transcranial Doppler sonography in patients with inadequate temporal bone windows. Ultrasound Med Biol, 24 , 101-103

Krabbe-Hartkamp MJ, van der Grond J, de Leeuw FE, de Groot JC, Algra A, Hillen B, Breteler MM, Mali WP (1998): Circle of Willis: morphologic variation on three-dimensional time-of-flight MR angiograms. Radiology, 207, 103-111

Kremser C, Lutz W, Auer A, Schmiedauer C, Felber S, Hochmair E, Aichner F: Transcranial Doppler ultrasonography: stereotactically guided examinations using magnetic resonance angiography. Biomed Tech, $\underline{44}, 265-271$

Lang J: Gehirn- und Augenschädel. Springer, Berlin 1979.

Lewis PM, Goldschlager T, Rosenfeld JV (2010): Image guidance for rapid temporal acoustic window localisation prior to transcranial Doppler ultrasound in the neurosurgical patient. $\mathrm{Br} \mathbf{J}$ Neurosurg, 24, 303-305

Lumenta C: Neurosurgery. Springer, Berlin 2008.

Maciunas RJ (2006): Computer-assisted neurosurgery. Clin Neurosurg, 53, 267-271 
Martin PJ, Evans D H, Naylor AR (1995). Measurement of blood flow velocity in the basal cerebral circulation: advantages of transcranial color-coded sonography over conventional transcranial Doppler. J Clin Ultrasound, 23, 21-26

Mascott CR (2006). In vivo accuracy of image guidance performed using optical tracking and optimized registration. J Neurosurg, $\underline{105}, 561-567$

Mathiesen T, Peredo I, Edner G, Kihlström L, Svensson M, Ulfarsson E, Andersson T (2007): Neuronavigation for arteriovenous malformation surgery by intraoperative three-dimensional ultrasound angiography. Neurosurgery, $\underline{60}, 345-350$

Muacevic A, Uhl E, Steiger HJ, Reulen HJ (2000): Accuracy and clinical applicability of a passive marker based frameless neuronavigation system. J Clin Neurosci, 7, 414-418

Nicoletto HA, Burkman MH (2009): Transcranial Doppler series part II: performing a transcranial Doppler. Am J Electroneurodiagnostic Technol, 49, 14-27

Rigamonti A, Ackery A, Baker AJ (2008): Transcranial Doppler monitoring in subarachnoid hemorrhage: a critical tool in critical care. Can J Anaesth, 55, 112-123

Ringel F, Ingerl D, Ott S, Meyer B (2009): VarioGuide: a new frameless image-guided stereotactic system--accuracy study and clinical assessment. Neurosurgery, $\underline{64}, 365-371$

Roberts DW, Strohbehn JW, Hatch JF, Murray W, Kettenberger H (1986): A frameless stereotaxic integration of computerized tomographic imaging and the operating microscope. J. Neurosurg, $\underline{65}, 545-549$

Seidel G, Kaps M, Gerriets T (1995): Potential and limitations of transcranial color-coded sonography in stroke patients. Stroke, 26, 2061-2066

Singh V, McCartney JP, Hemphill JC (2001): Transcranial Doppler ultrasonography in the neurologic intensive care unit. Neurol India, 49 Suppl 1, 81-89

Totaro R, Del Sette M, Marini C (1999): Echocontrast agents in neurosonology. Funct Neurol, $14,235-239$

Tsivgoulis G, Alexandrov Andrei V, Sloan MA (2009). Advances in transcranial Doppler ultrasonography. Curr Neurol Neurosci Rep, $\underline{9}$, 46-54 
van Gijn J, Kerr RS, Rinkel GJE (2007): Subarachnoid haemorrhage. Lancet, $\underline{369}$, 306-318

von Reutern GM (1982): Thoughts on training in ultrasound diagnosis of brain-supplying arteries. Ultraschall Med, $\underline{3}, 58-61$

von Reutern GM, Büdingen HJ: Ultraschalldiagnostik der hirnversorgenden Arterien. 2. Auflage; Thieme, Stuttgart 1993, 160-174

Widder B: Transkranielle Doppler-Sonographie bei zerebrovaskulären Erkrankungen; in: Beiträge einer Arbeitstagung über Transkranielle Doppler-Sonographie, die im Sommer 1986 in Ulm stattfand. Springer, Berlin 1987

Widder B, Görtler M: Doppler- und Duplexsonographie der hirnversorgenden Arterien. 6. Auflage; Springer, Berlin 2004

Wijnhoud AD, Franckena M, van der Lugt A, Koudstaal PJ, Dippel EDWJ (2008): Inadequate acoustical temporal bone window in patients with a transient ischemic attack or minor stroke: role of skull thickness and bone density. Ultrasound Med Biol, 34, 923-929

Willinsky RA, Taylor SM, TerBrugge K, Farb RI, Tomlinson G, Montanera W (2003): Neurologic complications of cerebral angiography: prospective analysis of 2,899 procedures and review of the literature. Radiology, 227, 522-528

Yagita Y, Etani H, Handa N, Itoh T, Imuta N, Okamoto M, Matsumoto M, Kinoshita N, Nukada T (1996): Effect of transcranial Doppler intensity on successful recording in Japanese patients. Ultrasound Med Biol, 22, 701-705 


\section{Anhang / Publikationen}

\subsection{Image Guidance for Transcranial Doppler Ultrasonography}

Kantelhardt SR, Greke C, Keric N, Vollmer F, Thiemann I, Giese A

Veröffentlicht: Neurosurgery 2011, Volume 68, ons257-ons266

Objective: Transcrancial Doppler ultrasonography (TCD) is an important tool for noninvasive detection and monitoring of vasospasm and other pathological conditions of the intracranial vessels. Intraoperatively it has been applied for identification of feeder- and drainage vessels and to ensure preservation of blood flow. In the intraoperative setting it has frequently been combined with image guidance. Conceptually modern image guidance systems allow integration of CTA or MRA based image guidance and TCD.

Methods: Three patients who underwent CTA scanning for reasons not related to this study were examined by neuronavigated image-guided TCD. The Doppler probe was fitted with reflective markers and tracked by a comercially available Kolibri ${ }^{\mathrm{TM}}$ frameless image-guidance system.

Results: Image-guided TCD allowed identification of all mayor intracranial vessels. Unilateral acquisition of reliable Doppler signals for ICA, carotid T, ACM, ACM bifurcation and ACA required between $14 \pm 6$ min. Pre-registration of these targets and detection by neuronavigation alone shortened examination times significantly to $8 \pm 2 \mathrm{~min}$. Registering the optimal examination trajectories on the neuronavigational device and application of a navigational pilot software shortened times for repetive examination further to $4 \pm 1 \mathrm{~min}$, besides it ensured that the examination was done at the exactly same spot under the same angle as in previous examinations.

Conclusions: Image guidance can easily and efficiently be applied to TCD, it provides anatomical orientation and may help to standardize investigation protocols, define pathological vascular territories for repeat investigations and therefore reduce inter- 
investigator variations. Image guidance may also extend the use of TCD to situations of a pathological or variant vascular anatomy.

\title{
9.2 Image-guided transcranial Doppler sonography for monitoring of defined segments of intracranial arteries
}

\author{
Greke C*, Neulen A, *, Kantelhardt SR, Birkenmayer A, Vollmer FC, Thiemann I, Giese A \\ (* diese Autoren haben zu gleichem Teilen zur Erstellung der Publikation beigetragen)
}

Publikation eingereicht, Veröffentlichung ausstehend

Background. Transcranial Doppler sonography (TCD) is widely used for non-invasive detection and monitoring of cerebral vasospasm in neurointensive care. However, it is associated with the difficulty of secure vessel identification as well as inter- and intrainvestigator variabilities.

Objective. Reliability and reproducibility of image-guided TCD were investigated in the clinical setting of intensive care.

Methods. The Kolibri ${ }^{\mathrm{TM}}$ neuronavigation system (Brainlab AG, Feldkirchen, Germany) was used to track a hand-held Doppler-probe of a DWL Multi-Dop ${ }^{\circledR}$ Tdigital device (Compumedics Germany GmbH, Singen, Germany). A non-invasive landmark-based patient registration was performed. The distance between planned vascular target and optimal Doppler signal was evaluated to measure the precision of the method for (i.) pre-procedurally planed target-points and (ii.) intra-procedurally acquired trajectories in serial examinations. Furthermore precision of non-invasive patient registration was analyzed.

Results. Using image guidance a corresponding flow signal was recorded immediately in all cases for MCA $(n=29)$, in $81 \%$ for carotid-T $(n=27)$ and in $90 \%$ for basilar-tip $(n=29)$. Examination of 94 pre-planned targets resulted in a spatial accuracy of $2.64 \pm 1.15 \mathrm{~mm}$. 
Reproducibility during serial re-examinations showed a spatial deviation of targets $2.64 \pm 1.05 \mathrm{~mm}$ for MCA, $2.71 \pm 1.17 \mathrm{~mm}$ for carotid-T and $2.9 \pm 1.44 \mathrm{~mm}$ for basilar-tip. There was no evidence that investigation time resulted in inaccuracy of non-invasive patient registration.

Conclusions. Image-guided TCD allows examination in the clinical setting of intensive care with high intra- and inter-procedural reproducibility. It facilitates identification of specific vessel segments and generation of standardized examination protocols for serial examinations. 


\section{Danksagung}

Mein besonderer und herzlicher Dank gilt meinem Betreuer und Doktorvater, Prof. Dr. med. Alf Giese, der mich stets uneingeschränkt unterstützt, motiviert und beispiellos gefördert hat. Die Möglichkeiten und Perspektiven, die mir seit Überlassung des Themas gegeben wurden, waren außergewöhnlich und haben in den vergangenen zwei Jahren meinen Werdegang in einem äußerst positiven Sinn beeinflusst.

Des Weiteren bedanke ich mich bei Dr. med. Axel Neulen und allen weiteren Mitarbeitern der Abteilung für Neurochirurgie, die mir im Verlauf meiner Arbeit geholfen und mich unterstützt haben. Ebenso danke ich den Mitarbeitern der anästhesiologischen Intensivstation für die freundliche und reibungslose Zusammenarbeit.

Ein weiterer besonderer Dank gilt Herrn Ingmar Thiemann und Herrn Achim Birkenmayer. Ihre Unterstützung und die vielen hilfreichen Gespräche waren ein wichtiger Beitrag zur Durchführung der Arbeit.

Rieke Peppel danke ich für die liebevolle Durchsicht meiner Arbeit.

Dr. med. Hans-Joachim Böhringer danke ich für die motivierende Anregung, eine Doktorarbeit in der Neurochirurgie durchzuführen.

Nicht zuletzt danke ich allen Studienteilnehmern und freiwilligen Probanden für ihre Geduld und ihre Unterstützung. 